October, 1998

TAC-1998-029

\title{
Equation of motion of a classical scalar field with back reaction of produced particles.
}

\author{
A.D. Dolgov [?, S.H. Hansen\} \\ Teoretisk Astrofysik Center \\ Juliane Maries Vej 30, DK-2100, Copenhagen, Denmark
}

\begin{abstract}
In the one-loop approximation we derive the equation of motion for a classical scalar field $\varphi_{c}(t)$ with the back reaction of particle production included. Renormalization of mass and couplings of $\varphi_{c}$ is done explicitly. The equation is non-local in time, but can easily be treated perturbatively or numerically. For the weak trilinear coupling of the external field to the produced particles, the new equation gives the same solution as the familiar one with the $\Gamma \dot{\varphi}_{c}$ term. For a stronger coupling and other types of couplings the results are significantly different. The equation can be applied to the universe heating by the inflaton decay and to spontaneous baryogenesis.
\end{abstract}

PACS: 98.80.Cq, 14.80.-j, 95.30.Cq

\section{Introduction}

In the classical theory of particle production (see e.g. [1]) the external time-dependent field is assumed to be "fixed", i.e. not affected by the back reaction of the produced particles. Though it is a very good approximation for the situation that can be realized under laboratory conditions, it is not so in cosmology, when an external cosmic field decays and transfers energy to the produced particles. Usually the back reaction is described by an addition of the term $\Gamma \dot{\varphi}_{c}$ into the usual KleinGordon equation governing the evolution of a (spatially homogeneous, though not necessarily so)

\footnotetext{
${ }^{1}$ Also: ITEP, Bol. Cheremushkinskaya 25, Moscow 113259, Russia.

${ }^{2}$ e-mail: dolgov@tac.dk

${ }^{3}$ e-mail: sthansen@tac.dk
} 
classical scalar field:

$$
\ddot{\varphi}_{c}+3 H \dot{\varphi}_{c}+V^{\prime}\left(\varphi_{c}\right)=-\Gamma \dot{\varphi}_{c}
$$

where $H=\dot{a} / a$ is the Hubble parameter describing the expansion of the universe (this term is absent in flat space-time), $V\left(\varphi_{c}\right)$ is the potential of the field $\varphi_{c}$, the prime denotes the derivative with respect to the field $\varphi_{c}$, and $\Gamma>0$ is the decay width. One argument in favour of a modeling the back reaction in this way is that the solution of eq. (何), in the case of the simplest harmonic potential, $V\left(\varphi_{c}\right)=m^{2} \varphi_{c}^{2} / 2$, and for the trilinear coupling to the produced particles, $\mathcal{L}_{\text {int }} \sim \varphi_{c} \chi^{*} \chi$, has the form:

$$
\varphi_{c}(t)=\varphi_{0} \exp (-\Gamma t / 2) \cos \left(\sqrt{m^{2}-\Gamma^{2} / 4} t\right)
$$

which correctly describes the behaviour of $\varphi_{c}$ known from perturbation theory (it is assumed here and in what follows that the space-time is flat). However, it is easy to see that already in the case of more complicated potentials and couplings, e.g. for $V\left(\varphi_{c}\right)=\lambda_{4} \varphi_{c}^{4} / 4$ and/or $\mathcal{L}_{\text {int }} \sim \varphi_{c}^{2} \chi^{*} \chi$, this ansatz is not applicable. Moreover, different equations may have the same solutions and the coincidence of the solutions in special cases does not imply the equivalence of the theories, as we will see below in some examples.

In what follows we will derive the equation of motion for the classical homogeneous scalar field, $\varphi_{c}(t)$, for different kinds of interactions with the produced quantum particles and different forms of the potential, $V(\varphi)$. In fact, the back reaction term does not depend on the form of $V(\varphi)$, but is determined only by the form of the interaction of $\varphi_{c}$ with the produced particles. On the other hand, the particle production rate is sensitive to the form of the potential $V(\varphi)$. The calculations here are made in flat space-time, but it is straightforward to generalize them [2] to the case of the Friedman-Robertson-Walker metric, because the latter is conformally flat. The results can be applied to the universe heating by the decay of the inflaton field and to spontaneous baryogenesis. Our approach here is very close to that of ref. [2]. 


\section{$2 \quad$ Trilinear scalar coupling}

Let us first consider the simplest case of the trilinear coupling of a scalar field $\varphi$ (which below will be taken as a classical spatially homogeneous field, $\left.\varphi_{c}(t)\right)$ to the massless scalar quantum field $\chi$. The Lagrangian of the fields $\varphi$ and $\chi$ has the form:

$$
\mathcal{L}(\varphi, \chi)=\frac{1}{2}(\partial \varphi)^{2}-V(\varphi)+\frac{1}{2}(\partial \chi)^{2}+f \varphi \chi^{2}
$$

The corresponding exact quantum operator equations of motion can be written as:

$$
\begin{array}{r}
\ddot{\varphi}-\Delta \varphi+V^{\prime}(\varphi)=f \chi^{2}, \\
\partial^{2} \chi=2 f \varphi \chi .
\end{array}
$$

We will assume that there exists a classical homogeneous field $\varphi_{c}(t)=\langle\varphi\rangle$, where the brackets mean averaging over quantum vacuum state of operators $\varphi$ and $\chi$. The equation of motion for $\varphi_{c}$ can be obtained by the quantum averaging of eq. (荬) in the presence of the classical field $\varphi_{c}$ [2]:

$$
\left\langle\ddot{\varphi}+V^{\prime}(\varphi)\right\rangle=\left\langle f \chi^{2}\right\rangle
$$

Taking average of the l.h.s. is trivial, it reduces to the substitution $\langle\varphi\rangle=\varphi_{c}(t)$, and averaging of the r.h.s. can be done using the formal solution of eq. (5):

$$
\chi(x)=\chi_{0}(x)+2 f \int d^{4} y G^{R}(x, y) \varphi_{c}(y) \chi(y)
$$

where $G^{R}(x, y)$ is the retarded Green's function and $\chi_{0}$ is the free field operator. The latter is canonically quantized according to:

$$
\chi(x)=\int \frac{d^{3} k}{(2 \pi)^{3 / 2} \sqrt{2 k_{0}}}\left(a_{\vec{k}} e^{-i k x}+a_{\vec{k}}^{\dagger} e^{i k x}\right),
$$

where $a_{\vec{k}}$ and $a_{\vec{k}}^{\dagger}$ are annihilation and creation operators of momentum $\vec{k}$, obeying the commutation relations $\left[a_{\vec{k}}, a_{\vec{k}^{\prime}}^{\dagger}\right]=(2 \pi)^{3} 2 k_{0} \delta^{3}\left(\vec{k}-\overrightarrow{k^{\prime}}\right)$.

The contribution of expression (7) into the r.h.s. of eq. (6) gives zero result to the first order in $f$. To be more precise, the condition of vanishing of $\langle\chi \chi\rangle$ to the first order in $f$ corresponds to 
elimination of tadpole diagrams and is achieved by a shift of $\varphi_{c}$. To the second order in $f$ the contribution is given by:

$$
f\langle\chi \chi\rangle=4 f^{2} \int \frac{d^{3} k}{(2 \pi)^{3} 2 k_{0}} \int d^{4} y e^{-i k(x-y)} G^{R}(x, y) \varphi_{c}(y) .
$$

Using the retarded Greens function for massless scalars:

$$
G_{s}^{R}(r)=\frac{1}{4 \pi r} \delta(r-t)
$$

we can make almost all the integrations in eq. (9) and obtain the equation of motion of the classical scalar field, $\varphi_{c}$, with the r.h.s. which describes the back reaction of the produced quanta of scalar field, $\chi$, on the evolution of the field $\varphi_{c}$ :

$$
\ddot{\varphi}_{c}+V^{\prime}\left(\varphi_{c}\right)=\frac{f^{2}}{4 \pi^{2}} \int_{0}^{t-t_{i n}} \frac{d \tau}{\tau} \varphi_{c}(t-\tau)
$$

where $t_{i n}$ is an initial time, when the particle production was switched on (it is assumed that $\left.t>t_{\text {in }}\right)$. The result is valid for the coupling of $\varphi_{c}$ to $\chi$ given by expression (3). For other forms of couplings and for produced particles with non-zero spin the r.h.s. would have different forms (see below). The term describing the back reaction of the produced particles is determined by the form of the interaction Lagrangian of the field $\varphi_{c}$ with the produced quantum fields, and does not depend upon the potential $V\left(\varphi_{c}\right)$. This is in contrast to the naive description of particle production by the term $\Gamma \dot{\varphi}_{c}$ with a constant $\Gamma$, suggested in earlier literature. It may approximately describe the realistic situation only for the harmonic potential, $V=m^{2} \varphi^{2} / 2$. If one wants to mimic particle production for other types of the potential by a similar local term, one would immediately conclude that such a term must have different forms for different potentials.

The integral in the r.h.s. of eq. (11) is logarithmically divergent at $\tau \rightarrow 0$. This divergence is related to the mass renormalization and can be regularized in the following way. Let us separate the integral in the r.h.s. into two parts, one from 0 to some $t_{1}$ and the other from $t_{1}$ to $\left(t-t_{\text {in }}\right)$. In the first integral let us subtract and add $\varphi_{c}(t)$. We obtain:

$$
\begin{aligned}
& \ddot{\varphi}_{c}+V^{\prime}\left(\varphi_{c}\right)-\frac{f^{2}}{4 \pi^{2}} \varphi_{c} \ln \frac{t_{1}}{\epsilon} \\
& =\frac{f^{2}}{4 \pi^{2}} \int_{0}^{t_{1}} \frac{d \tau}{\tau}\left[\varphi_{c}(t-\tau)-\varphi_{c}(t)\right]+\frac{f^{2}}{4 \pi^{2}} \int_{t_{1}}^{t-t_{i n}} \frac{d \tau}{\tau} \varphi_{c}(t-\tau) .
\end{aligned}
$$


The integrals in the r.h.s. are now finite. The logarithmically infinite contribution in the l.h.s., related to the ultraviolet cut-off $\epsilon \rightarrow 0$, is taken out by the mass renormalization, so with a possible bare mass term in the potential, $V_{m_{0}}=m_{0}^{2} \varphi_{c}^{2} / 2$ (here $m_{0}$ is the bare mass of $\varphi_{c}$ ), we obtain:

$$
\begin{aligned}
& \ddot{\varphi}_{c}+m^{2}\left(t_{1}\right) \varphi_{c}+\left[V^{\prime}\left(\varphi_{c}\right)-V_{m}^{\prime}\left(\varphi_{c}\right)\right] \\
& =\frac{f^{2}}{4 \pi^{2}} \int_{0}^{t_{1}} \frac{d \tau}{\tau}\left[\varphi_{c}(t-\tau)-\varphi_{c}(t)\right]+\frac{f^{2}}{4 \pi^{2}} \int_{t_{1}}^{t-t_{i n}} \frac{d \tau}{\tau} \varphi_{c}(t-\tau),
\end{aligned}
$$

where $t_{1}$ is an arbitrary normalization point and the "running" mass is $m^{2}\left(t_{1}\right)=m^{2}\left(t_{2}\right)-$ $\left(f^{2} / 4 \pi^{2}\right) \ln \left(t_{1} / t_{2}\right)$. In eq. (13) we explicitly separated the massive part, $V_{m_{0}}$, in the potential, so that the term in the square brackets vanishes for the harmonic potential, $V(\varphi)=m_{0}^{2} \varphi^{2} / 2$

In the limit of a small coupling, $f$, and for the harmonic potential, equation (13) can be solved analytically [2]. In this limit its solution coincides with that of the naive eq. (11). We will look for the solution in the form:

$$
\varphi_{c}=A(t) \cdot \sin (m t)
$$

where A is a slowly varying function of $t$. Substituting this into eq. (13) we obtain:

$$
-2 \dot{A} m \cos (m t)=\frac{f^{2}}{4 \pi^{2}} \frac{\pi}{2} A \cos (m t)
$$

and correspondingly $A(t) \sim \exp (-\Gamma t / 2)$ with $\Gamma=f^{2} / 8 \pi m$. It is the correct decay width of $\varphi_{c} \rightarrow 2 \chi$. Thus we found, that in the limit of a weak coupling both equations (1) and (13) have the same solution, though the equations themselves are quite different.

For the case of the harmonic potential one can reduce equation (11) to a more familiar form by making the Fourier transform:

$$
\varphi_{c}(t)=\int_{-\infty}^{\infty} \frac{d \omega}{2 \pi} \exp (-i \omega t) \tilde{\varphi}_{c}(\omega)
$$

where the spectral function $\tilde{\varphi}_{c}(\omega)$ should be analytic in the complex upper half-plane of $\omega$ to ensure vanishing of $\varphi_{c}$ for $t<t_{\text {in }}$ (below we take $t_{\text {in }}=0$ ); if $\varphi_{c}=$ const $\neq 0$ for $t<0$, this constant value can simply be subtracted from $\varphi_{c}$. Assuming that $t_{1}$ is sufficiently small $\left(m t_{1} \ll 1\right)$, so that the integral from 0 to $t_{1}$ in the r.h.s. of eq. (12) or (13) can be neglected, we obtain:

$$
\tilde{\varphi}_{c}(\omega)\left(m_{0}^{2}-\frac{f^{2}}{4 \pi^{2}} \ln \frac{t_{1}}{\epsilon}-\omega^{2}\right)=\frac{f^{2}}{4 \pi^{2}} \int_{-\infty}^{\infty} d t e^{i \omega t} \int_{t_{1}}^{t} \frac{d \tau}{\tau} \varphi_{c}(t-\tau) .
$$


For a large $t$ the integral over $d \tau$ depends weakly upon the upper limit, so that it can be pushed to infinity and the integration over $t$ can be done explicitly:

$$
\tilde{\varphi}_{c}(\omega)\left(m_{0}^{2}-\frac{f^{2}}{4 \pi^{2}} \ln \frac{t_{1}}{\epsilon}-\omega^{2}\right)=\frac{f^{2}}{4 \pi^{2}} \tilde{\varphi}_{c} \int_{t_{1}}^{\infty} \frac{d \tau}{\tau}(\cos \omega \tau+i \sin \omega \tau) .
$$

The second integral in the r.h.s. is equal to $i m \Gamma \operatorname{sign}(\omega) \tilde{\varphi}_{c}(\omega)$, while the first one, logarithmically divergent in the lower limit, gives mass renormalization in the momentum space. With the account of this term the renormalized mass can be written as:

$$
m_{0}^{2}-\frac{f^{2}}{4 \pi^{2}} \ln \frac{t_{1}}{\epsilon}-\frac{f^{2}}{4 \pi^{2}} \int_{\omega t_{1}}^{\infty} \frac{d y}{y} \cos y=m_{0}^{2}-\frac{f^{2}}{4 \pi^{2}} \ln \frac{\Lambda}{\omega}+(\text { finite terms }) \equiv m_{r e n}^{2},
$$

where $\Lambda=1 / \epsilon$ is the ultraviolet cut-off. Finally we obtain:

$$
\tilde{\varphi}_{c}(\omega)\left[m_{r e n}^{2}-\omega^{2}-i m_{r e n} \Gamma \operatorname{sign}(\omega)\right]=0
$$

This is a simple linear equation in momentum space. A similar equation for an unstable particle moving in an external potential (gravitational field) has been derived in ref. [3]. Eq. (22]) is different from eq. (1) with the $\Gamma \dot{\varphi}_{c}$-term, but in the limit of small $\Gamma / m$ it has the same solution (2). With an increasing $\Gamma / m$ the solution of eq. (11) ceases to oscillate and turns into an exponentially decaying one. For an even larger ratio $\Gamma / m$, especially in the unphysical limit $\Gamma \gg m$, the solution of eq. (11) tends to a constant. The solutions of eq. (20) behaves differently. For any value of the ratio $\Gamma / m$ the solution of this equation has both oscillating and exponentially decreasing factors. This behavior is demonstrated by the numerical solution of eq. (13), see figs. 1. Recall that eq. (13) is equivalent to the algebraic one (20) for the Fourier transformed field amplitude, only in the limit of a large $m t$. In the general case eq. (12) or (13) should be solved numerically.

There is a subtle point related to the definition of the value of the renormalized mass. It is clear that $m_{r e n}$ in eq. (20) and $m$ in eq. (2) (correspondingly $m$ in $V\left(\varphi_{c}\right)$ in eq. (11)) are normalized at the same point and should be taken equal when one compares the solution of these equations. On the other hand, the value $m^{2}\left(t_{1}\right)$ in eq. (13) is different from $m_{r e n}^{2}$, because of a finite contribution to $m^{2}$ from the r.h.s. of this equation, and depends therefore on $f$. This explains the presence of the coefficient $\alpha \neq 1$ in the envelope $\exp (-\alpha \Gamma t / 2)$ to the numerical solution of eq. (13), presented in figs. 1. 
There is no simple way to find an analytic solution for more complicated potentials, when the non-perturbed equation for $\varphi_{c}(t)$ is non-linear. For the case when the potential is dominated by the quartic term, $V(\varphi)=\lambda_{4} \varphi^{4} / 4$, the equation can be written as:

$$
z^{\prime \prime}+z^{3}=h^{2} \int_{x_{1}}^{x} \frac{d y}{y} z(x-y)
$$

Here the effective coupling constant is $h^{2}=f^{2} /\left(4 \pi^{2} \lambda_{4} \varphi_{0}^{2}\right)$. We introduced the dimensionless quantities $x=\sqrt{\lambda}_{4} \varphi_{0} t$ and $z(x)=\varphi / \varphi_{0}$, where $\varphi_{0}$ is the characteristic magnitude of the field $\varphi$. Prime means differentiation with respect to $x$. The contribution to the integral from 0 to $x_{1}$ is neglected as in the previous case (but it can easily be taken into account). We will solve this equation for $\varphi(t=0)=\varphi_{0}$ and $\dot{\varphi}(t=0)=0$.

In the limit of $h=0$ this equation is solved in terms of Jacobi elliptic functions (see e.g. 画):

$$
z_{0}(x)=\operatorname{cn}(x, 1 / \sqrt{2})
$$

In what follows we will use the notation $\operatorname{cn}(x, 1 / \sqrt{2}) \equiv \operatorname{cn}(x)$. It is easy to find the first order corrections in $h^{2}$ in the limit of relatively small time, $h^{2} x \ll 1$. We will look for the solution in the form:

$$
z(x)=A(x) \operatorname{cn}[\beta(x)]
$$

where $A(x)$ is a slowly varying function of $x$ and $\beta^{\prime}(x)=A(x)$, which ensures vanishing of the lowest order terms in the 1.h.s. of eq. (21). The function $A(x)$ differs from the one used above by the constant factor $\varphi_{0}$. Substituting expression (23) into eq. (21) and neglecting the term proportional to $A^{\prime \prime}$ we obtain:

$$
3 A^{\prime} A \frac{d}{d \beta}[\operatorname{cn} \beta(x)]=h^{2} \int^{x} \frac{d y}{y} A(x-y) \operatorname{cn}[\beta(x-y)] .
$$

In the first order in $h^{2} x$ the solution is straightforward:

$$
A(x) \approx 1-0.62 h^{2} x \approx 1-0.016 f^{2}\left(\sqrt{\lambda_{4}} \varphi_{0}\right)^{-1} t
$$

Unfortunately we failed to find an analytic approximation to the asymptotic decrease of the amplitude of the oscillating field $\varphi$ at very large $x$, which characterizes the particle production rate at 
a large time. The problem is that $A(x) \rightarrow 0$ for $x \rightarrow \infty$ and $\beta(x) \rightarrow$ const. Correspondingly the decomposition of $z$ as a product of quickly and slowly varying factors becomes invalid. However, the numerical treatment of the problem is quite simple and straightforward. The r.h.s. of equation (21) gives a nonzero contribution into the mass of the field $\varphi$, as is easy to see a negative one, $\delta m^{2}<0$. Correspondingly the solution, found numerically, asymptotically tends to the nonzero value, $\varphi_{\text {fin }}^{2}=-\delta m^{2} / \lambda$. A counter-term $\delta m^{2} \varphi^{2} / 2$ can be added to the potential, $V(\varphi)$, to ensure vanishing of the renormalized mass. The equation is solved in this case too and, as expected, $\varphi \rightarrow 0$. The decrease of its amplitude at small $h^{2} x$ agrees quite well with the approximate result (25) as is seen in figs. 2.

In connection with the derivation of the equation of motion for the primary field $\varphi$, given above and in the following sections, a very important question may arise. We derived equation (13) perturbatively including only one-loop contribution into $\langle\chi \chi\rangle$. Could one go beyond perturbation theory using this equation? We do not have a rigorous answer to this question (see also the discussion in the last section). However, there are quite many examples, that an equation with a perturbative potential (for example the Schroedinger equation with Coulomb potential) permits to go beyond perturbation theory. Moreover, the fact that equations (11) and (13) are different even in perturbation theory have important implications [2, 5] e.g. for spontaneous baryogenesis [6, 7].

\section{$3 \quad$ Quartic scalar coupling}

The calculations are essentially the same for other types of coupling of $\varphi_{c}$ to quantum fields. For the interaction of the form $\mathcal{L}_{i n t}=\lambda_{2} \varphi_{c}^{2} \chi^{2}$ the equation of motion for $\varphi_{c}$ takes the form:

$$
\ddot{\varphi}_{c}+V^{\prime}\left(\varphi_{c}\right)=\frac{\lambda_{2}^{2}}{2 \pi^{2}} \varphi_{c}(t) \int_{0}^{t-t_{i n}} \frac{d \tau}{\tau} \varphi_{c}^{2}(t-\tau) .
$$

The logarithmic divergence at $\tau \rightarrow 0$ can be removed by renormalization of the coupling constant, $\lambda_{4}$, in the self-interaction potential, $V_{\lambda}\left(\varphi_{c}\right)=\lambda_{4} \varphi_{c}^{4} / 4$. It can be done exactly in the same way as it was done above for mass renormalization. We separate the integral in the r.h.s. of eq. (26) into two parts: from 0 to $t_{1}$ and from $t_{1}$ to $\left(t-t_{i n}\right)$. In the first integral we subtract and add $\varphi_{c}^{2}(t)$ and 
obtain:

$$
\begin{array}{r}
\ddot{\varphi}_{c}+\lambda_{4}\left(t_{1}\right) \varphi_{c}^{3}+\left[V^{\prime}\left(\varphi_{c}\right)-V_{\lambda}^{\prime}\left(\varphi_{c}\right)\right]=\frac{\lambda_{2}^{2}}{2 \pi^{2}} \varphi_{c}(t) \int_{0}^{t_{1}} \frac{d \tau}{\tau}\left[\varphi_{c}^{2}(t-\tau)-\varphi_{c}^{2}(t)\right] \\
+\frac{\lambda_{2}^{2}}{2 \pi^{2}} \varphi_{c}(t) \int_{t_{1}}^{t-t_{i n}} \frac{d \tau}{\tau} \varphi_{c}^{2}(t-\tau),
\end{array}
$$

where $\lambda_{4}\left(t_{1}\right)$ is the coupling constant of the $\varphi^{4}$ self-interaction, renormalized at the normalization point $t_{1}$, by the loop with the field $\chi, \lambda_{4}\left(t_{1}\right)=\lambda_{4}\left(t_{2}\right)-\left(\lambda_{2}^{2} / 2 \pi^{2}\right) \ln \left(t_{1} / t_{2}\right)$. The integrals in the r.h.s. are now finite. We assume that $t_{1}$ is small, $m t_{1}<1$, while the solutions will be taken in the limit $m t>1$. Here we neglect the loop with the field $\varphi$ itself or, in other words, neglect the self-production of $\varphi$-quanta by the classical field $\varphi_{c}$. This is physically sensible if $\lambda_{4}<\lambda_{2}$.

A perturbative solution in this case is slightly more complicated than that given in the previous section. We assume that the potential is dominated by the harmonic part, $V\left(\varphi_{c}\right) \approx m^{2} \varphi_{c}^{2} / 2$, and that the coupling constants are small, $\lambda_{4}<\lambda_{2}<1$. To the lowest order in the coupling constants the solution has the form:

$$
\varphi_{c}(t)=A(t) \cos (m t+\Phi)+A^{3}(t)\left[c_{1} t \sin (m t+\Phi)+c_{2} \sin (3 m t+3 \Phi)+c_{3} \cos (3 m t+3 \Phi)\right]
$$

where $\Phi$ is a constant phase determined by initial conditions. The slowly varying amplitude $A(t)$ and the coefficients $c_{j}$ are given by:

$$
\begin{gathered}
A(t)=A_{i n}\left[1+\frac{\lambda_{2}^{2}}{16 \pi} \frac{A_{i n}^{2}}{m}\left(t-t_{i n}\right)\right]^{-1 / 2}, \\
c_{1}=-\frac{3 \bar{\lambda}}{8 m}+\frac{\lambda_{2}^{2}}{4 \pi^{2} m} \int_{0}^{m t} \frac{d \eta}{\eta} \sin ^{2} \eta \\
c_{2}=-\lambda_{2}^{2} /\left(128 \pi m^{2}\right) \\
c_{3}=\bar{\lambda} /\left(32 m^{2}\right)
\end{gathered}
$$

where:

$$
\bar{\lambda}=\lambda_{4}-\frac{\lambda_{2}^{2}}{2 \pi^{2}} \int_{m t_{1}}^{\infty} \frac{d \eta}{\eta} \cos 2 \eta
$$


The term proportional to $c_{1}$ in equation (28) rises linearly with time (in addition $c_{1}$ itself rises as $\ln m t$ ). This linear rise is the well known resonance behavior associated with the equality of the eigen-frequency of the non-perturbed equation and the perturbative force, coming both from the term proportional to $\lambda_{4} \varphi_{c}^{3}$ and from the terms in the r.h.s. of eq. (27). Moreover, if $\lambda_{4}$ is non-negligible (to be more precise, the combination $\lambda_{4} A_{i n}^{2} / m^{2}$ ), then the term $\lambda_{4} \varphi_{0}^{2} \varphi_{1}$ (where $\varphi_{0}$ is the zero-order approximation and $\varphi_{1}$ is the first-order correction) would induce a parametric resonance and an exponential rise of $\varphi$. Of course these resonances do not have physical sense, because the original equation does not possess any instability. The "resonances" (both the usual and the parametric ones) are quickly turned off with rising time and the large time behavior remains non-resonant, as is confirmed by the numerical calculations. A resonance amplification of the boson production rate would take place if the accumulation of the produced bosons is taken into account. To do that one has to make quantum averaging of the equations of motion, not over the vacuum state, as is done here, but over the states with non-zero boson occupation number. It will be done in a subsequent work [8].

The amplitude of the field, $\varphi_{c}(t)$, decreases in accordance with expression (29). This decrease is much slower than the exponential decay of $\varphi_{c}(t)$ considered in the previous section. Correspondingly the efficiency of particle production in the case of the quartic coupling is much weaker than that induced by the trilinear coupling in the previous section. The usual description of the back reaction of particle production by the $\Gamma \dot{\varphi}_{c}$ term in this case is very far from reality. Correspondingly the approach of the earlier papers [9, 10] (see also the recent review [11]) should be modified. The results of the numerical solution of eq. (27) are presented in figs. 3. For a weak coupling, i.e. for $\lambda_{2}^{2} m^{2} / 2 \pi^{2} A_{\text {in }}^{2} \ll 1$, they agree quite well with the perturbative result (28). The fitting coefficient $\alpha \neq 1$ appears because of renormalization of the parameters entering the l.h.s. of eq. (27) due to the interaction term in the r.h.s., while the dependence on time remains the same as that given by eq. (29).

The case of the quartic potential, $V(\varphi)=\lambda_{4} \varphi^{4} / 4$, can be treated similarly to that in the previous section. The solution is searched for in the form (23), so that the l.h.s. of eq.(24) remains the same, 
but the r.h.s. becomes different:

$$
3 A^{\prime} A \frac{d}{d \beta} \operatorname{cn}[\beta(x)]=\frac{\lambda_{2}^{2}}{2 \pi^{2} \lambda_{4}} \operatorname{cn}[\beta(x)] \int^{x} \frac{d y}{y} A^{2}(x-y) \operatorname{cn}^{2}(\beta(x-y)),
$$

where $x=\sqrt{\lambda_{4}} \varphi_{0} t$.

The decrease of the amplitude of the oscillations is described by the expression:

$$
A(t) \approx \frac{1}{1+0.0085\left(\lambda_{2}^{2} / \lambda_{4}\right) \varphi_{0} t} .
$$

To obtain this result, only the terms $\cos (p x)$ and $\cos (3 p x)$ (with $p \approx 0.85$ ) in the Fourier decomposition of $\operatorname{cn}[\beta(x)]$ (see e.g. [4) were taken into account. Again this behavior agrees quite well with the numerical solution for small couplings, see figs. 4. As above the fitting parameter $\alpha \neq 1$ is induced by renormalization.

It is interesting to note, that for the quartic coupling considered in this section the oscillations

asymptotically decrease faster in the case of the $\varphi^{4}$-potential, eq. (34), than in the case of the $\varphi^{2}$-potential, eq. (29). Further, comparing figs. 1 and 2 with figs. 3 and 4 (and the approximate behaviour of the envelopes) one realizes, that for the trilinear coupling, $f \varphi \chi^{2}$, the scalar field, $\varphi$, disappears much faster than for the quartic coupling. This is what one would expect, since the probability of the reaction induced by the coupling $\lambda_{2} \varphi^{2} \chi^{2}$ is quadratic in the $\varphi$-number density, whereas for the trilinear coupling the probability is linear in the $\varphi$-number density.

\section{Coupling to fermions}

When the classical scalar field couples to quantum fermions the calculations are slightly more complicated, but still straightforward. We will consider the Lagrangian density where the classical scalar field, $\varphi_{c}$, couples to the spin $1 / 2$ field $\psi$ through the Yukawa coupling:

$$
\mathcal{L}(\varphi, \psi, \bar{\psi})=\frac{1}{2}(\partial \varphi)^{2}-V(\varphi)+i \bar{\psi} \gamma_{\mu} \partial^{\mu} \psi+g \bar{\psi} \psi \varphi
$$

where $\psi$ is quantized according to:

$$
\psi(x)=\int \frac{d^{3} p}{(2 \pi)^{3} \sqrt{2 E}} \sum_{s}\left(a_{p}^{s} u^{s}(p) e^{-i p x}+b_{p}^{s \dagger} v^{s}(p) e^{i p x}\right)
$$


where $a$ and $b^{\dagger}$ are annihilation and creation operators at momentum $p$ and spin $s$, obeying the anti-commutation relations:

$$
\left\{a_{p}^{r}, a_{q}^{s \dagger}\right\}=\left\{b_{p}^{r}, b_{q}^{s \dagger}\right\}=(2 \pi)^{3} \delta^{3}(\vec{p}-\vec{q}) \delta^{r s} .
$$

Summation over spins, which we need in what follows, is achieved with the usual relations:

$$
\sum u^{s}(p) \bar{u}^{s}(p)=\not p+m \text { and } \sum v^{s}(p) \bar{v}^{s}(p)=\not p-m
$$

The equations of motion are:

$$
\begin{array}{r}
\ddot{\varphi}-\Delta \varphi+V^{\prime}(\varphi)=-g \bar{\psi} \psi, \\
i \not \partial \psi+g \psi \varphi=0, \\
-i \partial_{\mu}\left(\bar{\psi} \gamma^{\mu}\right)+g \bar{\psi} \varphi=0 .
\end{array}
$$

In the background of the classical field $\varphi_{c}(t)$ eq. (37) is formally solved by:

$$
\psi(x)=\psi_{o}(x)+i g \int d^{4} y G_{f}^{R}(x, y) \psi(y) \varphi_{c}(y)
$$

where $\psi_{0}$ is an initially free fermion field and the retarded Green's function for fermions is given by:

$$
G_{f}^{R}(x, y)=i \int \frac{d^{4} p}{(2 \pi)^{4}} \frac{\not p+m}{p^{2}-m^{2}} e^{-i p \cdot(x-y)}
$$

Here we will consider the case of massless fermions, which is technically simpler, and take fermionic masses into account in a subsequent paper [8]. There are some subtleties in the calculations in comparison with the case considered above of production of massless scalars, especially regarding renormalization and regularization. However, the calculations can essentially be reduced to the scalar case. The fermionic Green's function is expressed through the scalar one as:

$$
G_{f}^{R}(x, y)=i \not_{x} G_{s}^{R}(x-y)
$$

The vacuum expectation value of the field operators in the r.h.s. of eq. (36) is equal to:

$$
g\langle\bar{\psi}(x) \psi(x)\rangle=g^{2} \int d^{4} y \varphi_{c}(y) \frac{\partial G_{s}^{R}(x-y)}{\partial x^{\mu}} \operatorname{Tr}\left\langle\bar{\psi}_{0}(x) \gamma_{\mu} \psi_{0}(x)\right\rangle
$$


Using the representation (35) and taking vacuum expectation value and spinor trace we obtain:

$$
g\langle\bar{\psi}(x) \psi(x)\rangle=4 g^{2} \int d^{4} y \varphi_{c}(y) \frac{\partial G_{s}^{R}(x-y)}{\partial x^{\mu}} \frac{\partial S(x-y)}{\partial x^{\mu}}
$$

where:

$$
S(x-y)=\int \frac{d^{3} k}{(2 \pi)^{3} 2 E} e^{i k(x-y)},
$$

is the same function (coming from vacuum averaging of free field operators) which enters the r.h.s. in the scalar case. It satisfies the condition:

$$
\partial^{2} S=0
$$

while the Green's function satisfies:

$$
\partial^{2} G(x-y)=\delta^{4}(x-y)
$$

Using these two equations and the identity:

$$
2 \partial_{\mu} S \partial_{\mu} G=\partial^{2}(S G)-G \partial^{2} S-S \partial^{2} G
$$

we obtain:

$$
\ddot{\varphi}_{c}+V^{\prime}\left(\varphi_{c}\right)=-\frac{g^{2}}{4 \pi^{2}} \frac{d^{2}}{d t^{2}} \int_{0}^{t-t_{i n}} \frac{d \tau}{\tau} \varphi_{c}(t-\tau)-g^{2} \Lambda^{2} \varphi_{c}
$$

The last term, proportional to the ultraviolet cut-off parameter, $\Lambda$, renormalizes the mass of $\varphi_{c}$. The term under the integral sign is the same as the one in the scalar case eq. (11) and its logarithmic divergence at $\tau=0$ can be treated in a similar way. However, instead of the term $\varphi_{c} \ln \left(t_{1} / \epsilon\right)$ we obtain $\ddot{\varphi}_{c} \ln \left(t_{1} / \epsilon\right)$. This corresponds to wave function renormalization (renormalization of the kinetic term) at the time moment $t_{1}$. Finally we obtain:

$$
\begin{aligned}
& {\left[1+\frac{g^{2}}{4 \pi^{2}} \ln \left(\frac{t_{1}}{\epsilon}\right)\right] \ddot{\varphi}_{c}+V_{r e n}^{\prime}\left(\varphi_{c}\right)} \\
& =-\frac{g^{2}}{4 \pi^{2}} \frac{d^{2}}{d t^{2}}\left\{\int_{0}^{t_{1}} \frac{d \tau}{\tau}\left[\varphi_{c}(t-\tau)-\varphi_{c}(t)\right]+\int_{t_{1}}^{t-t_{i n}} \frac{d \tau}{\tau} \varphi_{c}(t-\tau)\right\},
\end{aligned}
$$

where $V_{\text {ren }}\left(\varphi_{c}\right)$ is the potential of $\varphi_{c}$ with renormalized mass. 
The perturbative solution in the case of a harmonic potential, $V\left(\varphi_{c}\right)=m^{2} \varphi_{c}^{2} / 2$, is essentially the same as in section 2. If we assume $\varphi_{c}=A(t) \sin (m t)$, where $A(t)$ is a slowly varying function of $t$, we find that $\dot{A} / A=g^{2} m /(16 \pi)$. For a small $\Gamma$ it is essentially the same result as can be found from the naive ansatz with the $\Gamma \dot{\varphi}_{c}$ term in the r.h.s. of eq. (11) using $\Gamma_{\varphi_{c} \bar{\psi} \psi}=g^{2} m_{\varphi_{c}} /(8 \pi)$ [2]. For the harmonic potential, $V(\varphi)=m^{2} \varphi^{2} / 2$, and the Yukakwa coupling to fermions, $g \varphi \bar{\psi} \psi$, the particle production is quite similar to that considered in the first part of section 2. The results of the numerical calculations for this case are presented in figs. 5 .

For the quartic potential, $V(\varphi)=\lambda_{4} \varphi^{4} / 4$, we will look for the perturbative solution in the form (23). In direct analogy with the previous sections it is easy to find, that the amplitude $A(x)$ decays as:

$$
A(t) \approx \frac{\varphi_{0}}{1+0.011 g^{2} \sqrt{\lambda_{4}} \varphi_{0} t} .
$$

The results of the numerical calculations are presented in figs. 6 .

Now, one could also consider non-renormalizable couplings, such as $\mathcal{L}_{\text {int }}=\kappa \varphi^{2} \bar{\psi} \psi$, where $\kappa$ has dimension (mass $)^{-1}$. The corresponding equation of motion has the form:

$$
\ddot{\varphi}_{c}+V^{\prime}\left(\varphi_{c}\right)=-\frac{\kappa^{2}}{2 \pi^{2}} \varphi(t) \frac{d^{2}}{d t^{2}} \int_{0}^{t-t_{i n}} \frac{d \tau}{\tau} \varphi_{c}(t-\tau)^{2} .
$$

An approximate analytic solution for the potential $V(\varphi)=m^{2} \varphi^{2} / 2$ can be found along the same lines as presented in the beginning of section 3. The essential part of the solution behaves as:

$$
\varphi(t)=\varphi_{0}\left(1+\frac{\varphi_{0}^{2} \kappa^{2}}{4 \pi} m t\right)^{-1 / 2} \cos m t .
$$

This result describes the numerical solution quite well (see figs. 7).

If the potential is dominated by the $\lambda_{4} \varphi^{4}$-term, eq. (50) can be solved with the ansatz (23). The amplitude decays as:

$$
A(t)=\left(1+0.075 \sqrt{\lambda_{4}} \kappa^{2} \varphi_{0}^{3} t\right)^{-1 / 3}
$$

The decay is astonishingly slow. It can be compared with the numerical solutions presented in figs. 8. The agreement is quite good if the above mentioned effect of renormalization is taken into account. 
The numerical solution of eq. (50) can be compared to the solution of eq. (48) and one sees that, as in the bosonic case, the scalar field, $\varphi(t)$, falls off much faster for the coupling $g \varphi \bar{\psi} \psi$ than for $\kappa \varphi^{2} \bar{\psi} \psi$. The reason being, that the latter goes quadratically with the number density.

\section{Discussion and conclusion}

We have seen that with a weakly coupled external field, $\varphi_{c}(t)$, the effect of particle production on its evolution can be described by the addition of an extra term into the usual Klein-Gordon equation. This extra term is given by an integral over all previous history of the field, $\varphi_{c}(t)$, and the concrete form of the integrand is determined by the form of the interaction of $\varphi_{c}$ with the produced particles (see examples considered above). In all the cases it has been assumed that the interaction is bilinear in terms of the produced quantum fields:

$$
\mathcal{L}_{i n t}=F_{b}(\varphi) \chi^{*} \chi+F_{f}(\varphi) \bar{\psi} \psi
$$

where $\chi$ and $\psi$ are bosonic and fermionic quantum fields respectively. The calculations have been done in the limit of a negligible mass of the produced particles and in flat space-time. Lifting both restrictions is straightforward but tedious and will be considered elsewhere [8].

The most essential restriction is the weakness of the field, $\varphi_{c}(t)$, such that the Green's functions of the produced particles can be taken as the free field ones. This assumption is justified if the effective mass of the produced quanta, generated by the interaction (53), is small in comparison with their characteristic energy. The latter is essentially the frequency of the oscillations of $\varphi_{c}(t)$. In this approximation our equation adequately describes the decrease of the amplitude of $\varphi_{c}(t)$ induced by the particle production.

Another simplifying assumption, which was made above, is that the number density of the produced particles is small, so that neither Fermi-Dirac suppression nor Bose-Einstein enhancement is essential. Correspondingly the production of fermions would be further suppressed in comparison with our results, while the production of bosons could be quite significantly enhanced. The effect of quantum statistics is expected to give rise to a resonance amplification of the boson production 
and will be considered elsewhere. Finally we assume, that $\lambda_{4}$ in the potential is so small that we can ignore self-production of $\phi$-quanta by the field $\varphi_{c}$.

The case of a strong external field is considerably more complicated. There is no closed expression for the Green's function of the produced particles in a strong external field and no closed equation for $\varphi_{c}(t)$ can be written down. To overcome this problem one may use an adiabatic approach. In the case of the bilinear coupling to the produced particles (53) the calculation of the particle production rate can be reduced to the solution of an ordinary differential equation with timedependent frequency (see e.g. [1]). It is all done in the "fixed" field approximation i.e. without field decay due to particle production. The corresponding decrease of the field amplitude can be taken into account adiabatically by imposing conservation of the total energy. In the historically first papers [12, 13, 14] on particle production by the inflaton field, the process was considered perturbatively in a spirit close to the present work, but without inclusion of the effect of backreaction of the produced particles on the evolution of the inflaton field.

The non-perturbative calculations, valid for an arbitrary strongly coupled field, $\varphi_{c}(t)$, were first undertaken in refs. [15, 16]. In both papers the possibility of an enhanced production of bosons due to parametric resonance excitation was noticed, but it was argued [15] that parametric resonance is not operative, in the framework of the model considered there, because of the cosmological expansion and rescattering of the produced particles. It has later been found that the resonance may be wide and the effects of particles leaving the resonance mode is not so important, permitting parametric enhancement of boson production by the inflaton decay (see ref. [17]).

Non-perturbative production of fermions was considered in ref. [15] for the usual Yukawa coupling to fermions, $g \varphi \bar{\psi} \psi$, and for an arbitrary time dependence of the scalar field, $\varphi_{c}(t)$. Concrete examples were given for the case of harmonic oscillations of $\varphi$, which take place if $\varphi$ "live" in the potential, $V(\varphi)=m^{2} \varphi^{2} / 2$, and the back reaction of particle production on $\varphi$-evolution is neglected. It was shown there that in the weak field limit the rate of particle production is described by a constant decay width, $\Gamma$, in accordance with perturbation theory. In the limit of a strong field the production probability goes down as $1 / \varphi_{0}^{1 / 2}$, where $\varphi_{0}$ is the amplitude of the oscillations of the field $\varphi(t)$. This 
suppression is related to an increase of the effective mass of the produced fermions, $m_{e f f} \sim g \varphi_{c}$, while the frequency of the oscillations of $\varphi$ remains constant. This effect, of course, cannot be traced in the present work, where the weak field approximation is used.

In a recent paper [18] a non-peturbative approach has been applied to fermion production by the field $\varphi_{c}(t)$ with the potential $m^{2} \varphi^{2} / 2+\lambda \varphi^{4} / 4$ !. A system of coupled equations for the evolution of the field, $\varphi_{c}$, and the mode functions of the produced fermions was solved numerically in one loop approximation. This permitted to take the back reaction of particle production on $\varphi_{c}$-evolution into account. However, our equation differs from that derived in ref. 18] in the weak field limit (linear in the field $\varphi_{c}$ ). This difference can possibly be ascribed to a difference in our approaches to renormalization and the initial time singularity. As is seen, from the arguments presented here, we treat the initial time singularity as a usual ultraviolet singularity which can be regularized by the standard renormalization procedure. In ref. [18] this singularity was avoided with the help of a Bogoliubov transformation. In the limit of a large field strength, such that the effective mass of fermions, $g \varphi_{c}$, is larger than the mass of the field $\varphi_{c}$, the calculations of ref. 118] showed a strong suppression of particle production in agreement with ref. [15]. However, it is found there that the suppression persists even in the case of $g \varphi_{c} \ll m_{\varphi}$, though not for extremely small $g \varphi_{c}$. This result is in contradiction with the calculations of ref. [15], according to which the production rate in this region of parameters is well described by perturbation theory.

Recently the approach of the paper [15] was repeated in ref. [19] for the particular case of particle production by the scalar field in the potential $\lambda \phi^{4}$. It was found that the suppression of the particle production at large values of the field amplitude for the case of harmonic potential, as observed in [15], does not take place for $V=\lambda \phi^{4} / 4$, and the production of fermions may be quite efficient. This conclusion was also made (a little earlier) in ref. [18]. Possible explanations of the deviation of these results, from the results of ref. [15], is an influence of the $\lambda \varphi^{4}$-term in the potential and corresponding excitation of higher modes, as well as an increase of the oscillation frequency of $\varphi_{c}$ due to a bigger amplitude of $\varphi$.

Returning to the results of the present paper, we repeat that they are valid in the weak field 
approximation and, as such, can be applied to the universe heating by the inflaton decay at the final stage of the inflationary process, when the amplitude of the inflaton field is sufficiently small. Even though the inflaton energy during this period only contributes a relatively small fraction of the initial inflaton energy, its role in the creation of the matter in the universe could be much more significant. The reason for that is that at the final stage of the decay, when the potential is approximately $m^{2} \varphi_{c}^{2} / 2$, the inflaton behaves as non-relativistic matter. Therefore its energy density is amplified, with respect to the energy of the relativistic particles produced earlier, by the redshift factor $a(t) / a_{i n}(t)$. This makes the role of the final part of the inflaton decay correspondingly enhanced and the temperature at the end of the decay becomes close to the lowest order perturbative result [20]. Another mechanism of suppression of particle production was discussed in ref. [21], where it was shown that parametric amplification might be effectively suppressed by the final state interaction of the produced particles.

Another possible application of our results is spontaneous baryogenesis. The evolution of the spatially homogeneous pseudo-goldstone field, $\theta$, which is related to spontaneous breaking of baryonic charge conservation, is described by the equation:

$$
f^{2} \ddot{\theta}+U^{\prime}(\theta)=\frac{d}{d t} J_{B}^{0}
$$

where $J_{B}^{0}$ is the baryonic charge density and $f$ is the scale of spontaneous symmetry breaking. Since, as we have shown, the impact of particle production on the evolution of $\varphi_{c}$ is not given by the term $\Gamma \dot{\varphi}$, it means that the naive identification $f^{2} \Gamma \dot{\theta}=d J_{B}^{0} / d t$ is not correct. Correspondingly the baryon asymmetry is not given by $\delta J_{B}^{0} \sim \Gamma \delta \theta$ (for a more detailed discussion see refs. [2, 5]).

Acknowledgment. The work of $\mathrm{AD}$ and $\mathrm{SH}$ was supported in part by the Danish National Science Research Council through grant 11-9640-1 and in part by Danmarks Grundforskningsfond through its support of the Theoretical Astrophysical Center. AD is greatful to the Scuola Normale Superiore (Pisa) where a part of this work has been done.

\section{References}


[1] A.A. Grib, S.G. Mamaev, and V.M. Mostepanenko, "Vakuumnye kvantovye effekty v silnykh polyakh", Energoatomizdat, Moskva, 1988 (in Russian); "Vacuum Quantum Effects in Strong Fields", Friedmann Laboratory Publishing, St.Petersburg, 1994 (in English).

[2] A. Dolgov and K. Freese, Phys. Rev. D 51 (1995) 2693.

[3] A.D. Dolgov, ZhETF, 79 (1980) 337; Phys.Rev., D 24 (1981) 1042.

[4] I.S. Gradshtein and I.M. Ryzhik, "Tables of Integrals, Series, and Products", Academic Press Inc. 1994.

[5] A. Dolgov, K. Freese, R. Rangarajan, and M. Srednicki, Phys. Rev. D , 56 (1997) 6155.

[6] A. Cohen and D. Kaplan, Phys. Lett. B199 (1987) 251; Nucl. Phys. B 308 (1988) 913.

[7] A.D. Dolgov, Surveys in High Energy Physics, 13 (1998) 83; hep-ph/9707419.

[8] A.D. Dolgov and S.H. Hansen, in preparation.

[9] M.S. Turner, Phys. Rev. D 28 (1983) 1243.

[10] R.J. Scherrer and M.S. Turner, Phys. Rev. D 31 (1985) 681.

[11] G. Lazarides, hep-ph/9802415.

[12] A.D. Dolgov and A.D. Linde, Phys. Lett. B116 (1982) 329.

[13] L.F. Abbott, E. Farhi, and M.B. Wise, Phys. Lett. B117 (1982) 29.

[14] A. Albrecht, P.J. Steinhardt, M.S. Turner and F. Wilczek, Phys. Rev. Lett. 48 (1982) 1437.

[15] A.D. Dolgov and D.P. Kirilova, Yad. Fiz. 51 (1990) 273; English translation: Sov. J. Nucl. Phys. 51 (1990) 172.

[16] J.H. Traschen and R.H. Brandenberger, Phys. Rev. D 42 (1990) 2491.

[17] A partial list of references include: L. Kofman, A. Linde, and A. Starobinsky, Phys. Rev. Lett. 73 (1994) 3195; Y.Shtanov, J.Traschen and R.Brandenberger, Phys. Rev. D 51 (1995) 5438. D. 
Boyanovsky, M. D’attanasio, H.J. de Vega, R. Holman and D.-S.Lee; Phys. Rev. D 52 (1995) 6805; D. Kaiser, Phys. Rev. D 53 (1996) 1776; D. Boyanovsky, H.J. de Vega, R. Holman and J.F.J. Salgado, Phys. Rev. D 54 (1996) 7570; S.Yu. Khlebnikov and I.I. Tkachev, Phys. Rev. Lett. 77 (1996) 219; L. Kofman, A. Linde, and A. Starobinsky, Phys. Rev. D 56 (1997) 3258; P.B. Greene, L. Kofman, A. Linde and A.A. Starobinsky, Phys. Rev. D 56 (1997) 6175; L. Kofman, Rel. astr. phys.: Novikov's 60th Birthday. Conf., Cambridge Uni. Press 1997, p. 133;

[18] J. Baacke, K. Heitmann and C. Pätzold, hep-ph/9806205.

[19] P. B. Green and L. Kofman, hep-ph/9807339.

[20] H. Fujisaki, K. Kumekawa, M. Yamaguchi, and M. Yoshimura, Phys. Rev. D 54 (1996) 2494; M. Yoshimura, hep-ph/9605246.

[21] R. Allahverdi, B.A. Campbell, Phys. Lett. B395 (1997) 169. 


\section{Figure Captions:}

Fig. 1 Trilinear scalar coupling, $f \varphi \chi^{2}$, and harmonic potential, $V=m^{2} \phi^{2} / 2$. The decrease of the amplitude is given by the envelope function, $\exp (-\alpha \Gamma t / 2), \Gamma=f^{2} / 8 \pi m$. Fig. 1a: $m=1, f=$ $0.7, \lambda=0, \alpha=1.03$. Fig. 1b: $m=1, f=1.4, \lambda=0, \alpha=1.25$.

Fig. 2 Trilinear scalar coupling, $f \varphi \chi^{2}$, and potential, $V=\lambda \phi^{4} / 4$. The decrease of the amplitude is given by the envelope function, $1-0.016 \alpha f^{2}\left(t-t_{i n}\right)$. Fig. $2 \mathrm{a}: m_{r}=0, f=0.5, \lambda=1, \alpha=0.94$. Fig. 2b: $m_{r}=0, f=1.0, \lambda=1, \alpha=0.92$.

Fig. 3 Quartic scalar coupling, $\lambda_{2} \varphi^{2} \chi^{2}$, and harmonic potential, $V=m^{2} \phi^{2} / 2$. The decrease of the amplitude is given by the envelope function, $\varphi_{0}\left(1+\alpha \lambda_{2}^{2} /(16 \pi) \varphi_{0}^{2} / m\left(t-t_{i n}\right)\right)^{-1 / 2}$. Fig. 3a: $m=1, \lambda_{2}=0.5, \lambda_{4}=0, \alpha=1$. Fig. 3b: $m=1, \lambda_{2}=1.5, \lambda_{4}=0, \alpha=1.5$.

Fig. 4 Quartic scalar coupling, $\lambda_{2} \varphi^{2} \chi^{2}$, and potential, $V=\lambda_{4} \phi^{4} / 4$. The decrease of the amplitude is given by the envelope function, $\varphi_{0} /\left(1+0.085 \alpha \lambda_{2}^{2} / \lambda_{4} \varphi_{0}\left(t-t_{i n}\right)\right)$. Fig. 4a: $m_{r}=$ $0, \lambda_{2}=0.5, \lambda_{4}=1, \alpha=1.05$. Fig. $4 \mathrm{~b}: m_{r}=0, \lambda_{2}=1.0, \lambda_{4}=1, \alpha=1.3$.

Fig. 5 Trilinear fermion coupling, $g \bar{\psi} \psi \varphi$, and harmonic potential, $V=m^{2} \phi^{2} / 2$. The decrease of the amplitude is given by the envelope function, $\exp (-\alpha \Gamma t / 2), \Gamma=g^{2} m /(8 \pi)$. Fig. $5 \mathrm{a}: m=$ $1, g=0.5, \lambda=0, \alpha=1.0$. Fig. 5b: $m=1, g=1.0, \lambda=0, \alpha=0.8$.

Fig. 6 Trilinear fermion coupling, $g \bar{\psi} \psi \varphi$, and potential, $V=\lambda_{4} \phi^{4} / 4$. The decrease of the amplitude is given by the envelope function, $\varphi_{0} /\left(1+0.011 \alpha g^{2} \sqrt{\lambda_{4}} \varphi_{0}\left(t-t_{\text {in }}\right)\right)$. Fig. 6a: $m=0, g=$ $0.5, \lambda=1, \alpha=1.0$. Fig. $6 \mathrm{~b}: m=0, g=1.0, \lambda=1, \alpha=0.82$.

Fig. 7 Quartic fermion coupling, $\kappa \varphi^{2} \bar{\psi} \psi$, and harmonic potential, $V=m^{2} \phi^{2} / 2$. The decrease of the amplitude is given by the envelope function, $\left[1+\alpha \varphi_{0}^{2} \kappa^{2} m t /(4 \pi)\right]^{-1 / 2}$. Fig. $7 \mathrm{a}: m=1, \kappa=$ $0.5, \lambda=0, \alpha=1.0$. Fig. $7 \mathrm{~b}: m=1, \kappa=1.0, \lambda=0, \alpha=1.0$.

Fig. 8 Quartic fermion coupling, $\kappa \varphi^{2} \bar{\psi} \psi$, and potential, $V=\lambda_{4} \phi^{4} / 4$. The decrease of the amplitude is given by the envelope function, $\left(1+0.075 \alpha \sqrt{\lambda_{4}} \kappa^{2} \varphi_{0}^{3} t\right)^{-1 / 3}$. Fig. 8a: $m=0, \kappa=$ $0.5, \lambda=1, \alpha=1.0$. Fig. 8b: $m=0, \kappa=1.0, \lambda=1, \alpha=0.9$. 


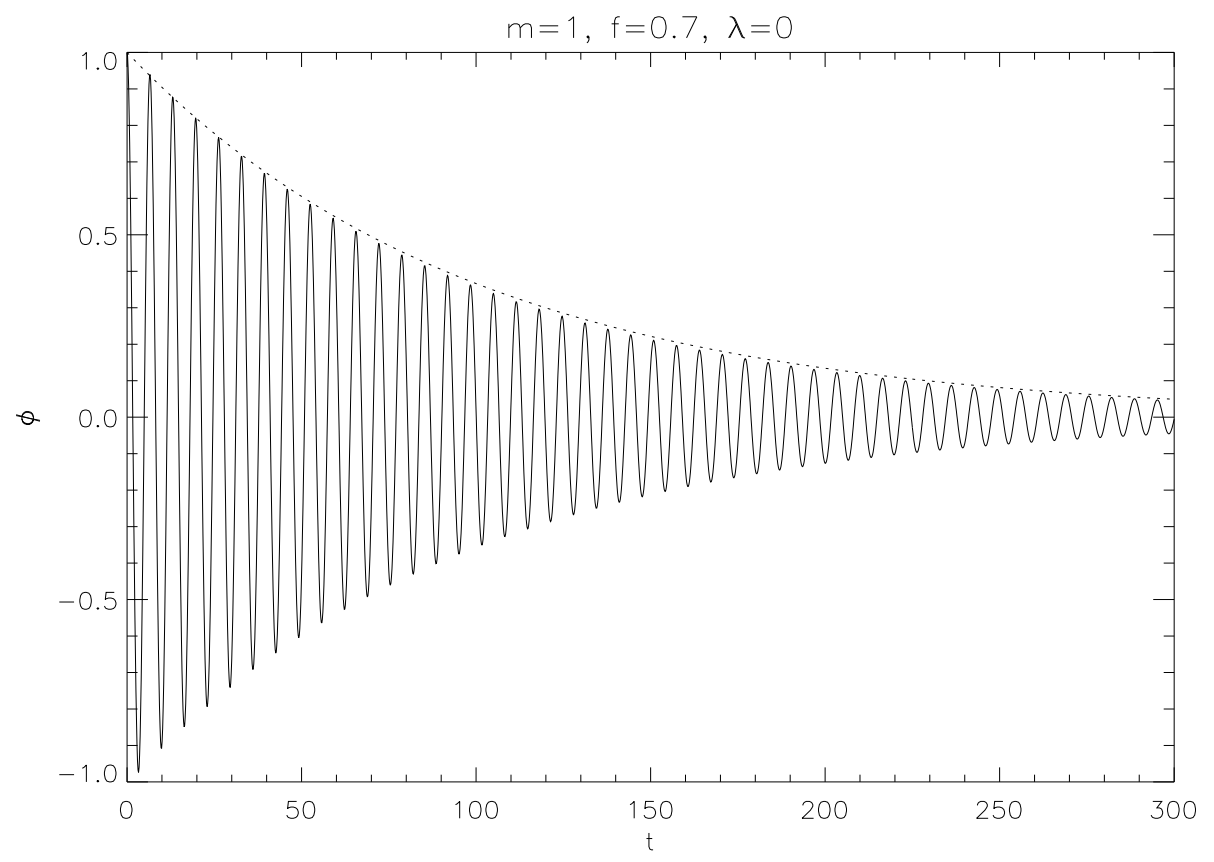

Figure 1a.

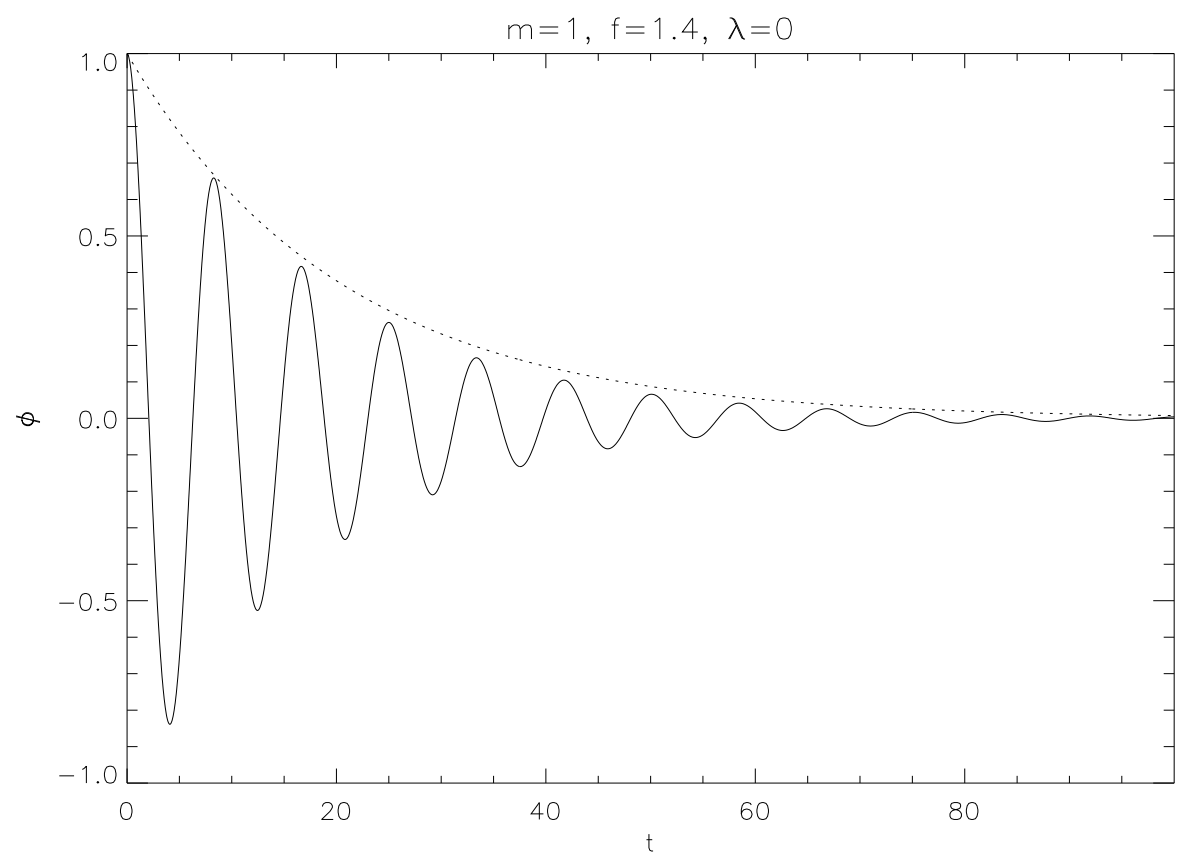

Figure 1b. 


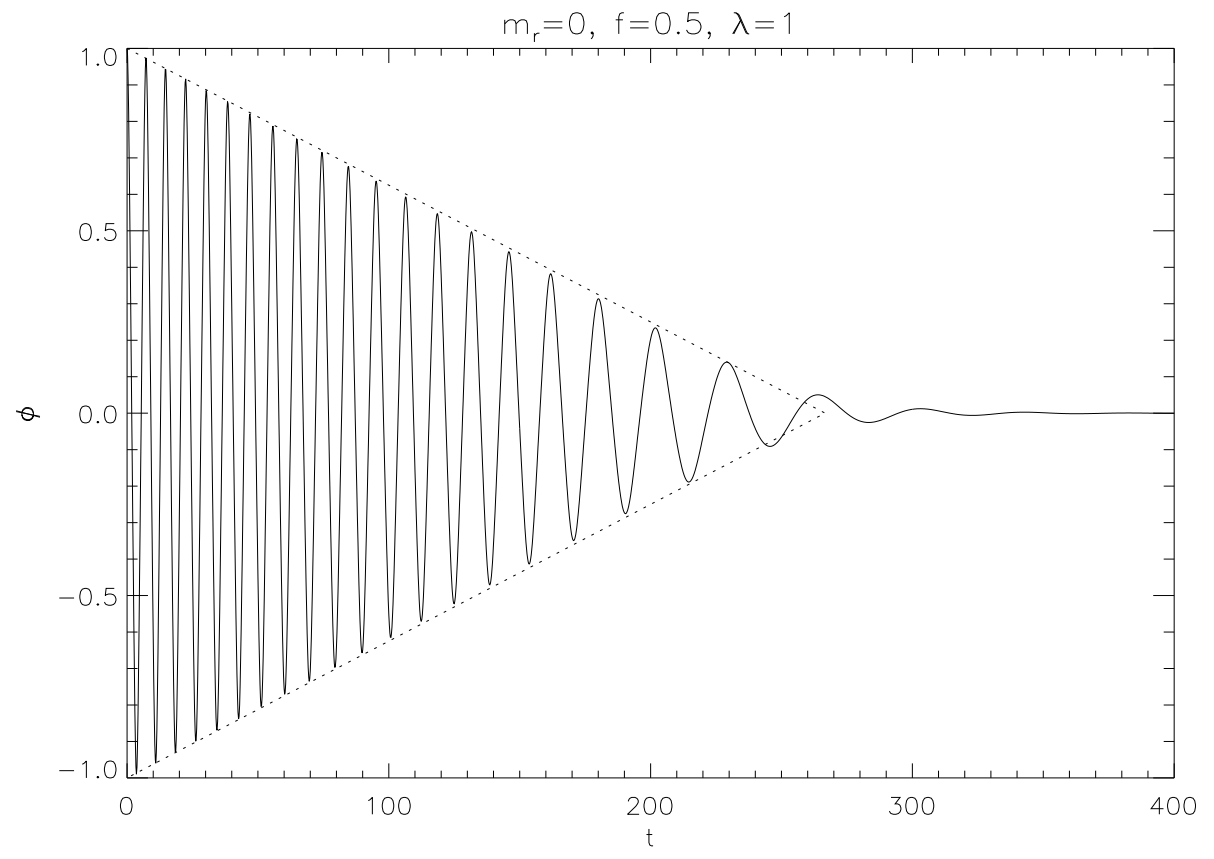

Figure 2a.

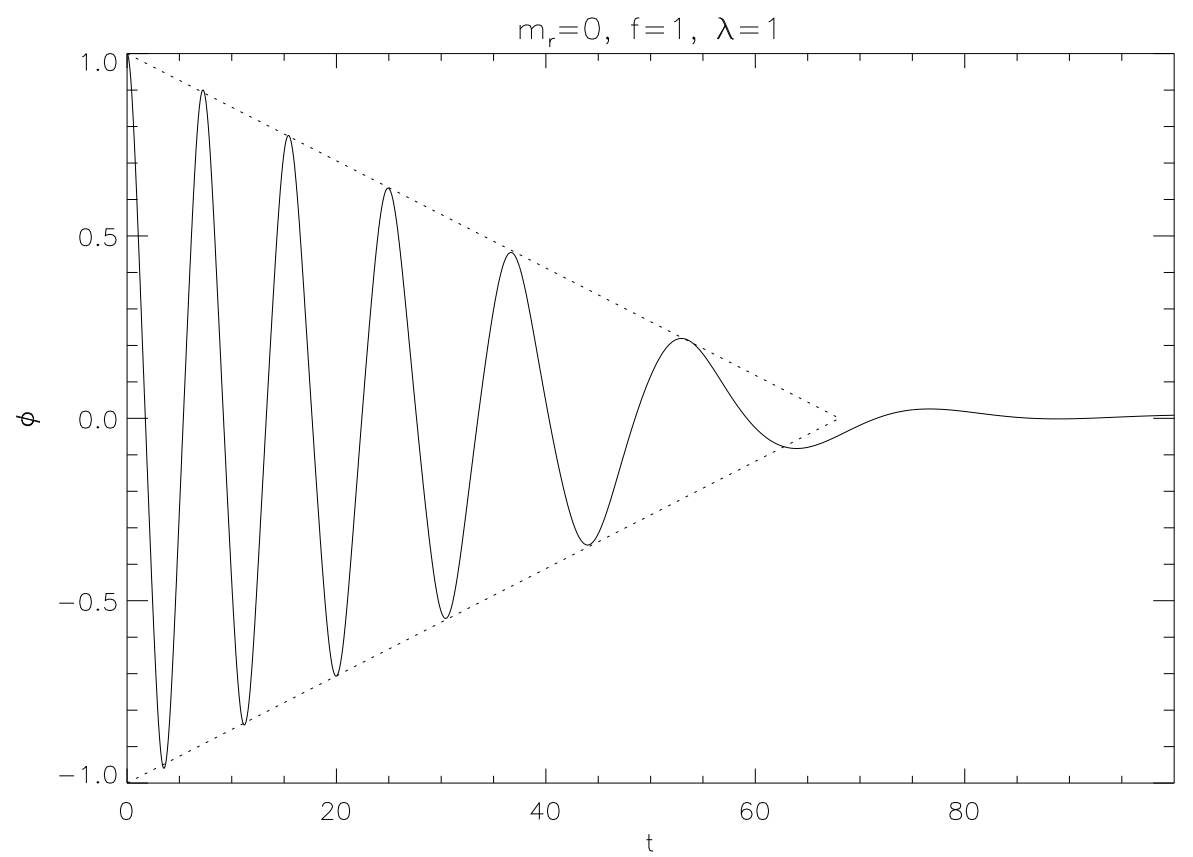

Figure 2b. 


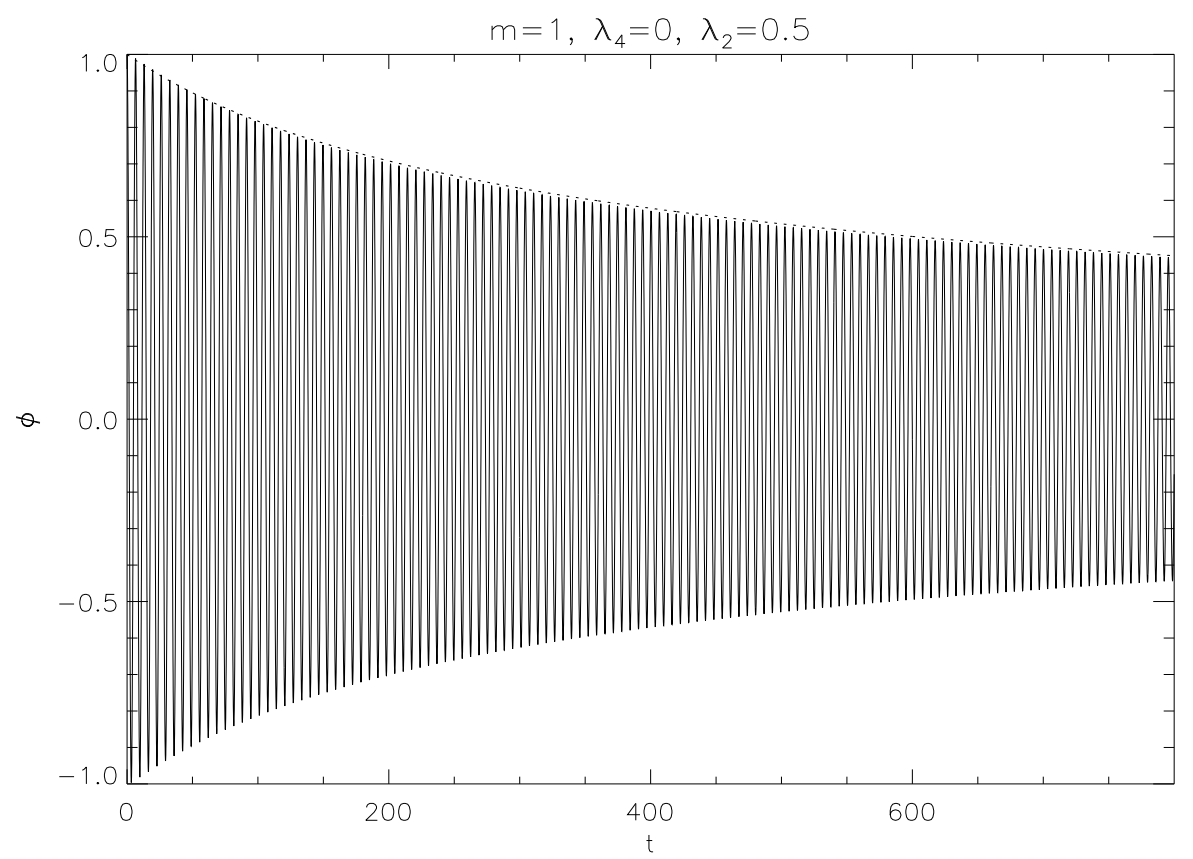

Figure 3a.

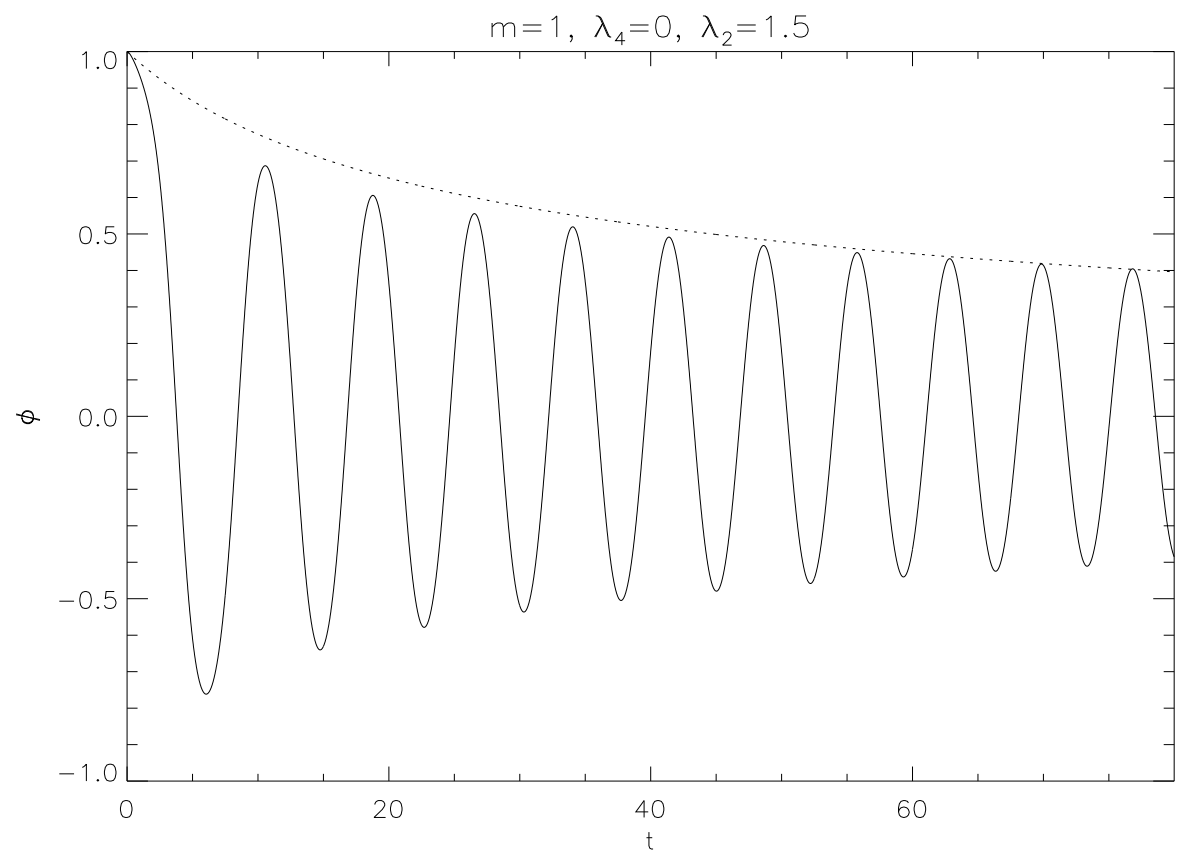

Figure 3b. 


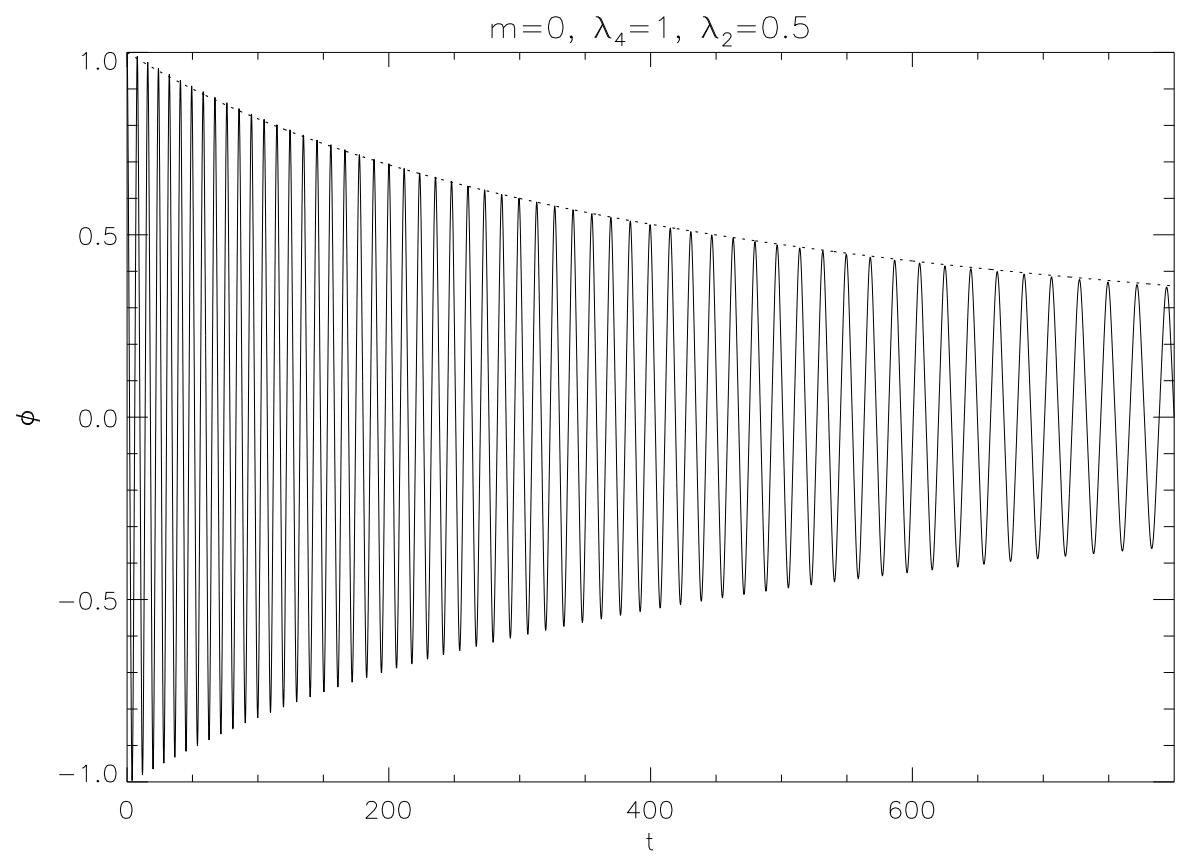

Figure 4a.

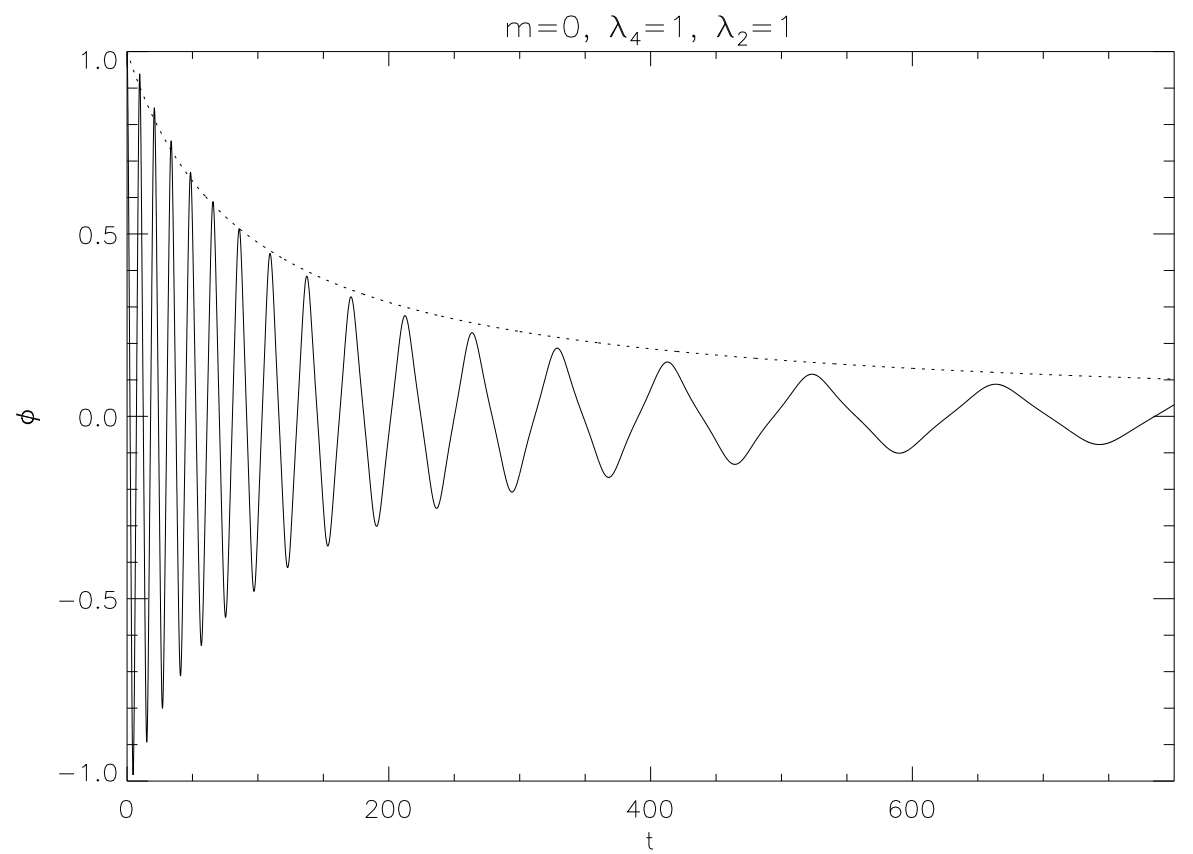

Figure $4 \mathrm{~b}$. 


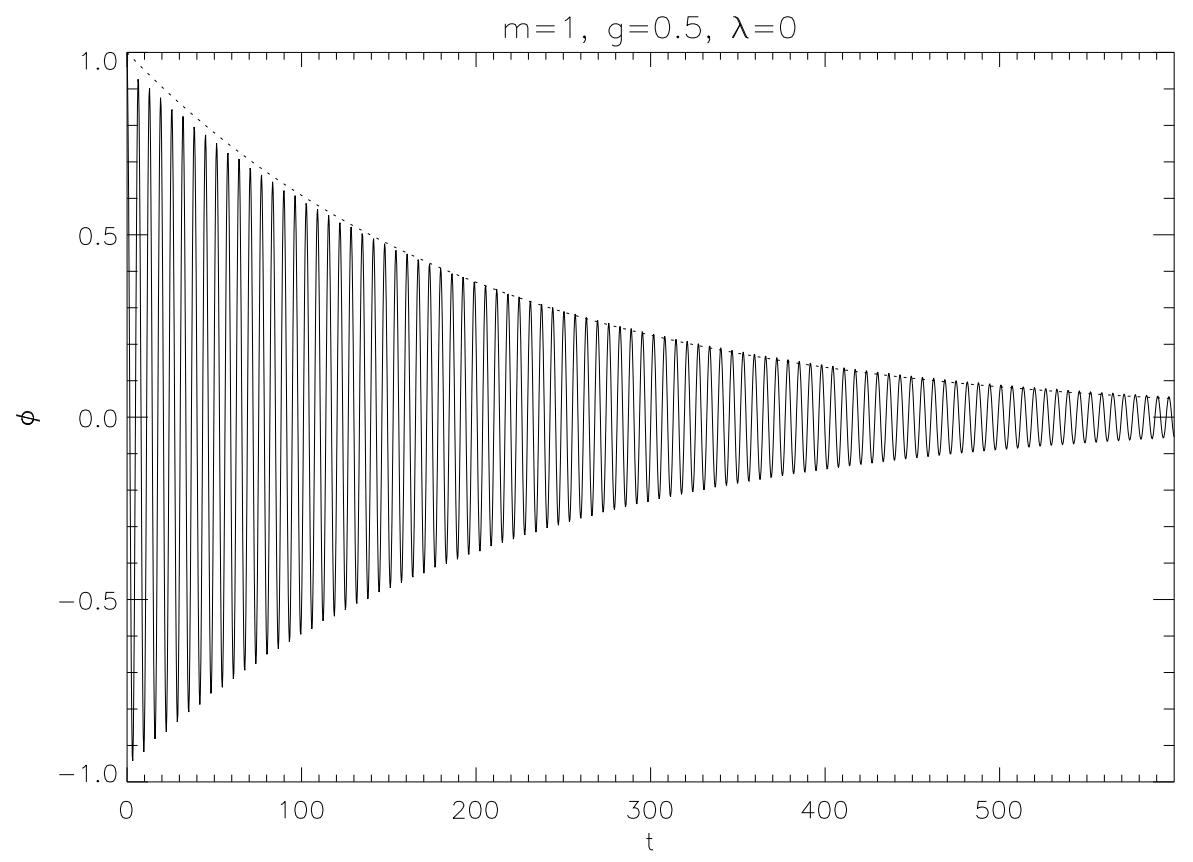

Figure 5a.

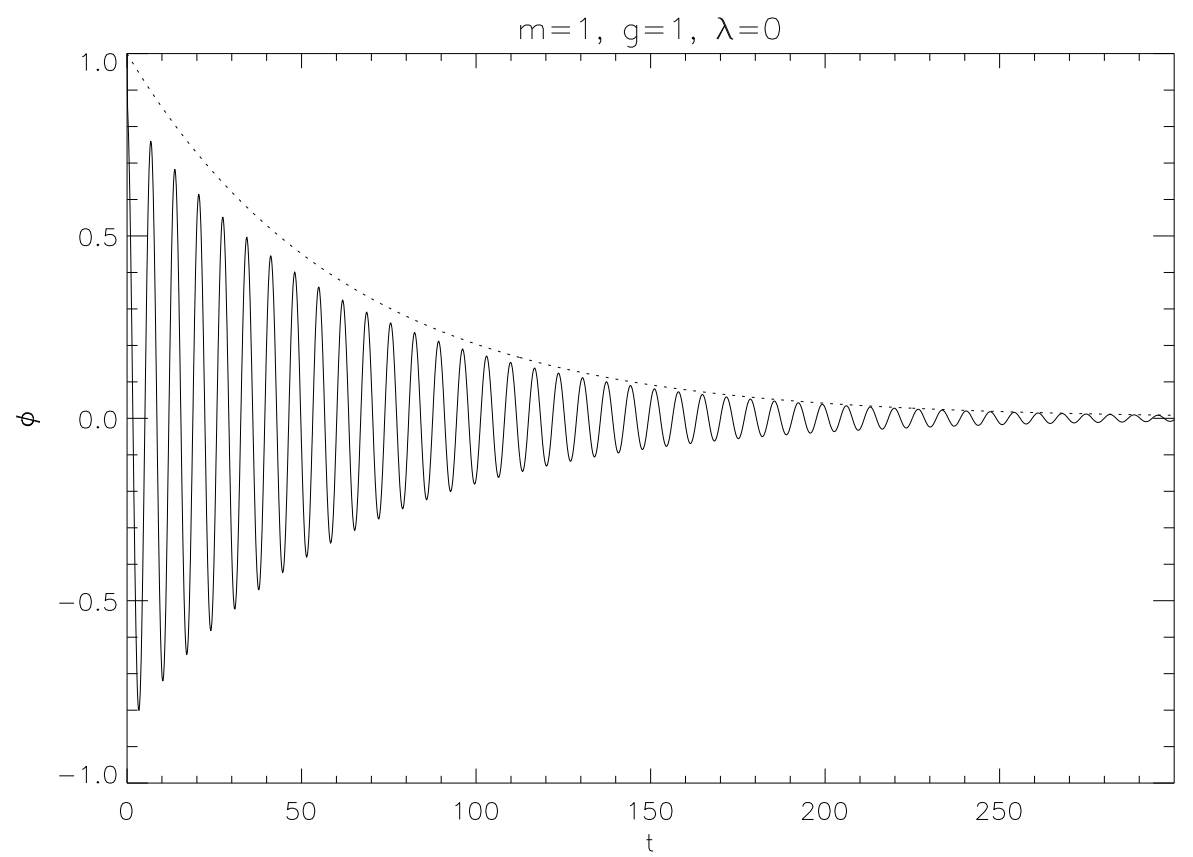

Figure 5b. 


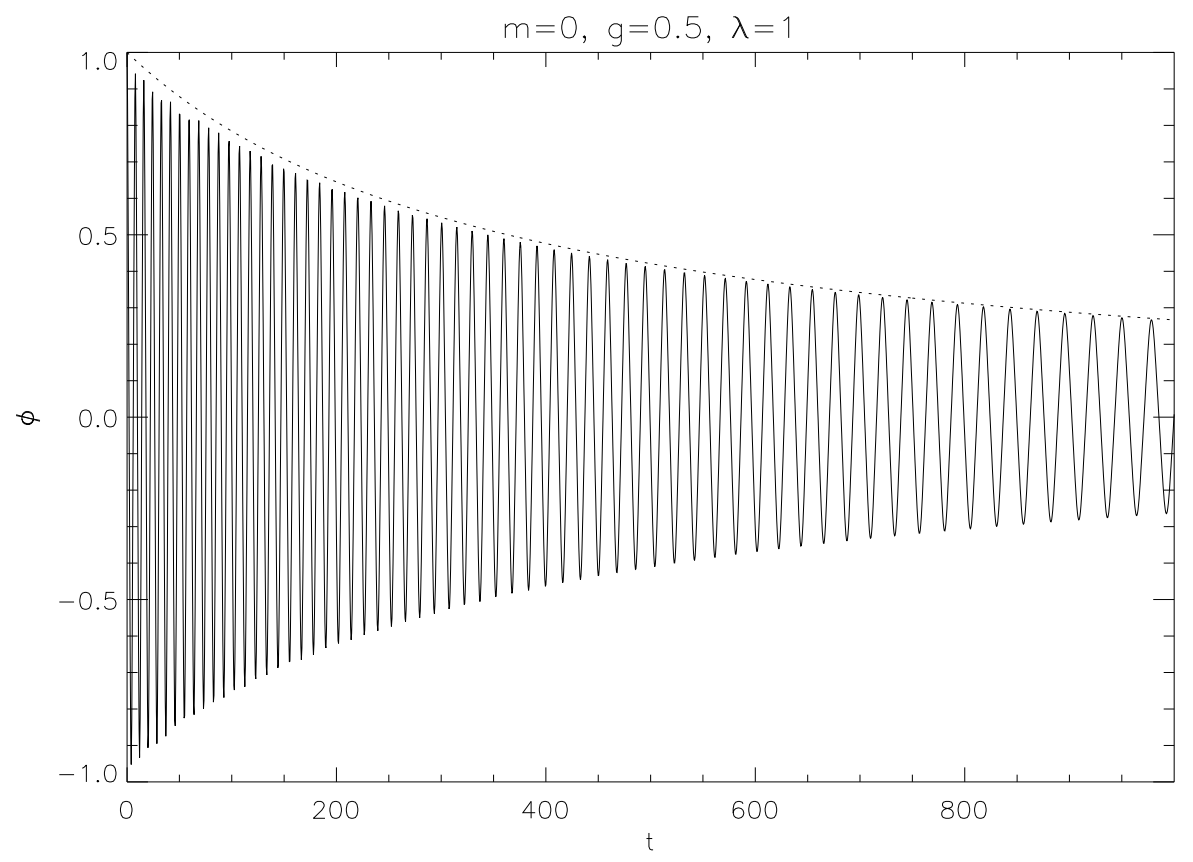

Figure 6a.

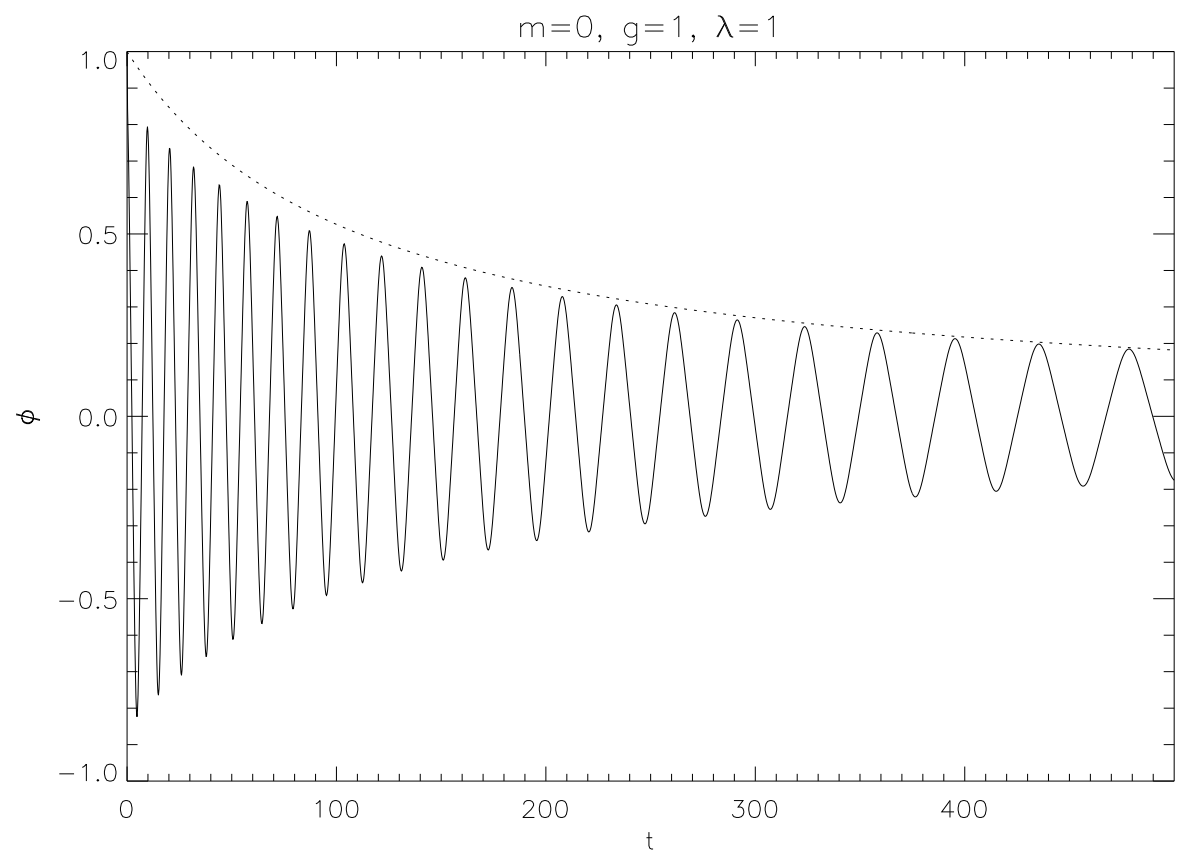

Figure $6 \mathrm{~b}$. 


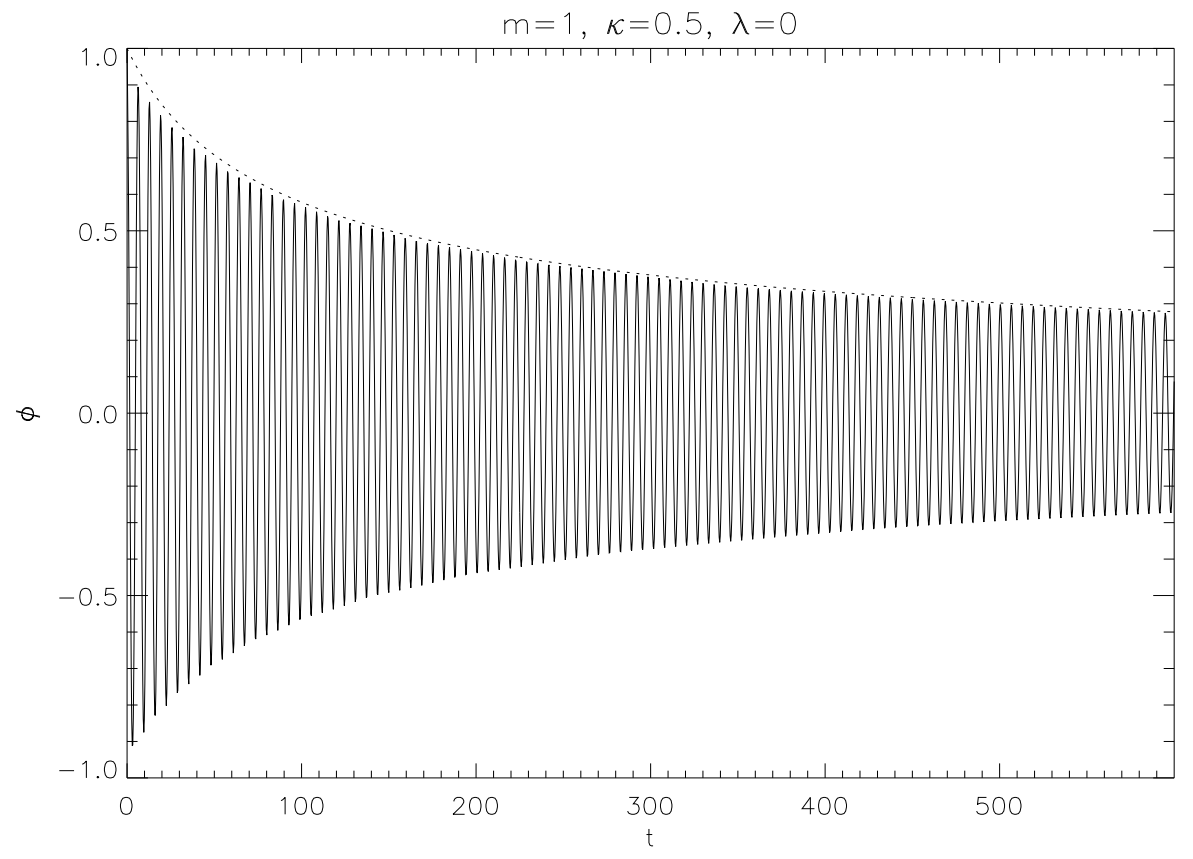

Figure 7a.

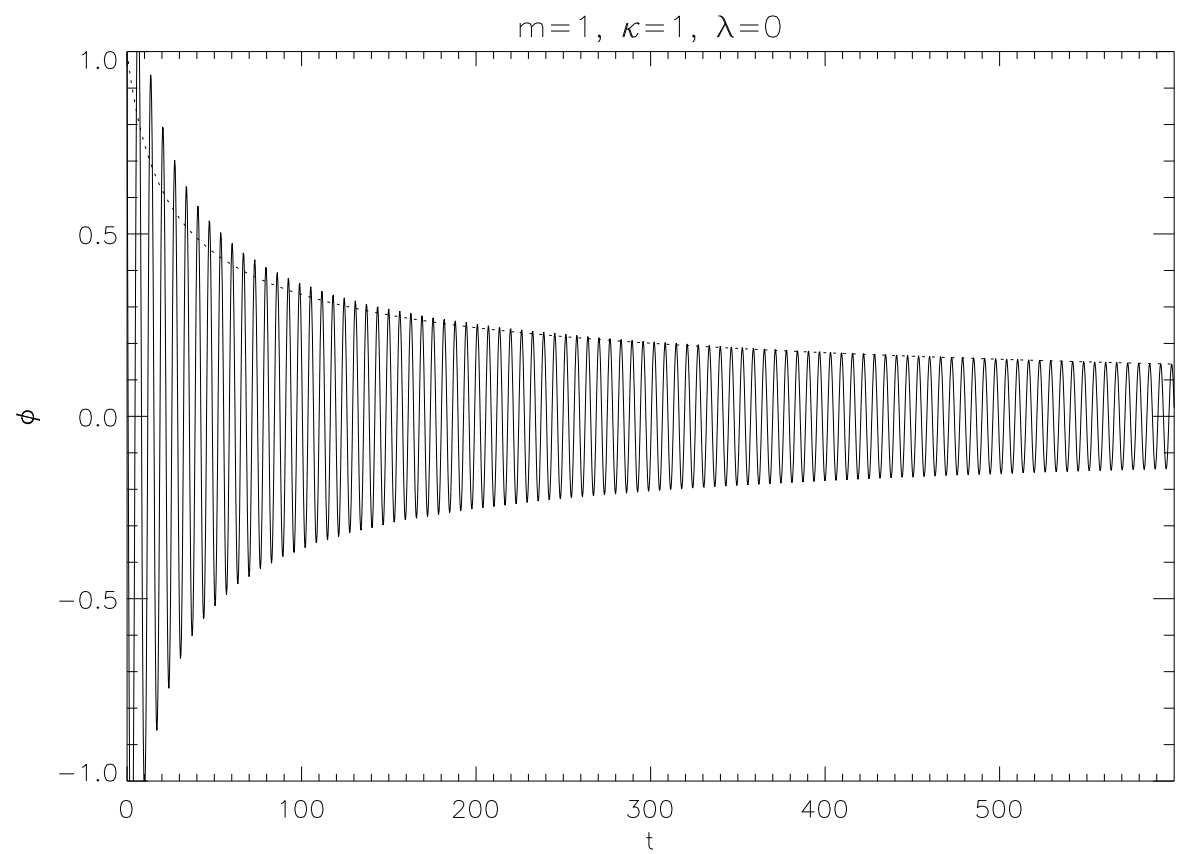

Figure 7b. 


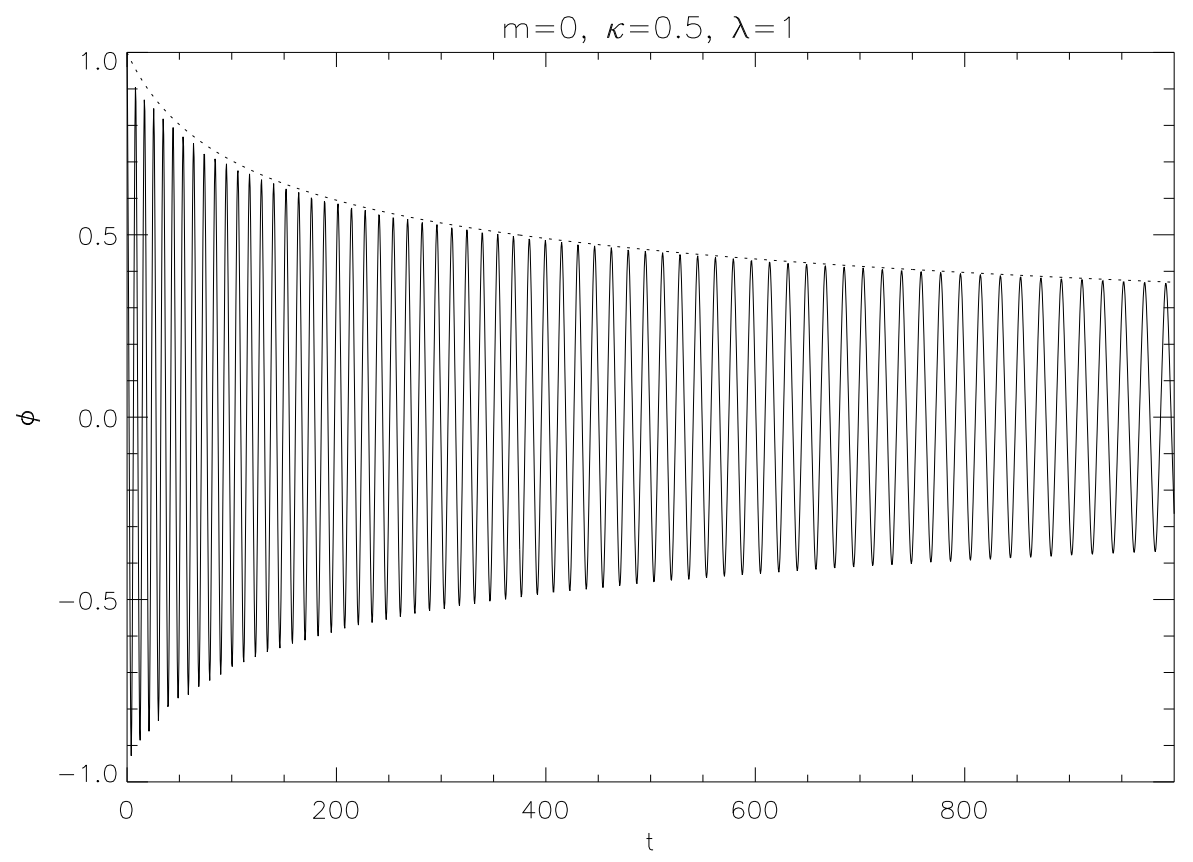

Figure 8a.

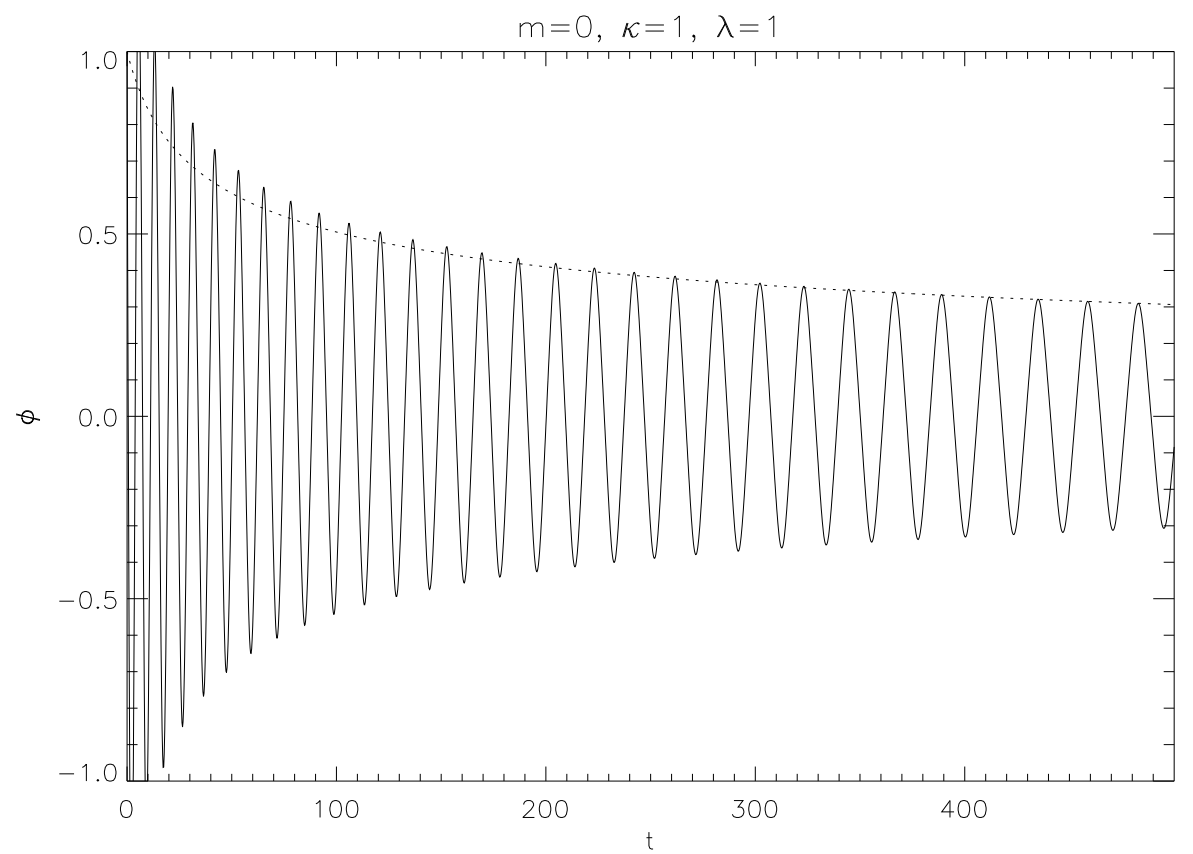

Figure 8b. 